\title{
An overview of the two-phase solvent systems used in the countercurrent separation of phenylethanoid glycosides and iridoids and their biological relevance
}

\author{
Simon Vlad Luca • Anca Miron · Svetlana Ignatova • \\ Krystyna Skalicka-Woźniak (10)
}

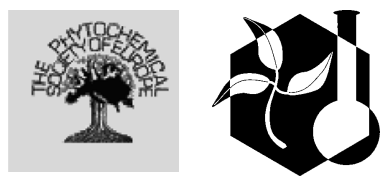

Received: 8 April 2018/Accepted: 31 January 2019/Published online: 20 February 2019

(C) The Author(s) 2019

\begin{abstract}
Phenylethanoid glycosides (PhGs) and iridoids are two vast groups of water soluble secondary metabolites widely distributed in plant kingdom and well known for their important biological activities. Their purification by conventional chromatography is time consuming, uses large amounts of organic solvents and requires repeated steps. Moreover, the sample recovery is low, because the hydroxyl groups of PhGs and iridoids make them strongly adsorbed onto the solid support (silica gel, Sephadex LH-20) during separation. Being a liquid-liquid based technology, countercurrent separation (CCS) comes as an alternative tool to overcome the issues associated
\end{abstract}

Electronic supplementary material The online version of this article (https://doi.org/10.1007/s11101-019-09599-y) contains supplementary material, which is available to authorized users.

S. V. Luca · A. Miron

Department of Pharmacognosy, Grigore T. Popa

University of Medicine and Pharmacy Iasi, 16

Universitatii Street, 700115 Iasi, Romania

S. V. Luca · K. Skalicka-Woźniak $(\bowtie)$

Department of Pharmacognosy with Medicinal Plant Unit,

Medical University of Lublin, 1 Chodzki Street,

20-093 Lublin, Poland

e-mail: kskalicka@pharmacognosy.org

S. Ignatova

Department of Chemical Engineering, Advanced Bioprocessing Centre, CEDPS, Brunel University

London, Kingston Lane, Uxbridge UB8 3PH, UK with solid-phase adsorbents. It has been successfully used for the separation of many groups of specialized plant metabolites. This is the first extensive review describing the application of CCS for purification of iridoids and $\mathrm{PhGs}$, based on the research papers from the last 20 years (1998-2018) that used countercurrent chromatography and centrifugal partition chromatography technologies. In total, 65 papers described the isolation of 84 different phytochemicals (28 PhGs and 56 iridoids) from 42 plant species belonging to 16 distinct families that were separated with 59 different biphasic solvent systems. Since PhGs and iridoids are highly polar molecules, more than half of the employed systems (56\%) were composed of various ratios of ethyl acetate-alcohol-water, as well as binary systems, such as ethyl acetate-water and $n$-butanolwater. The current review may be used as starting point for CCS users on their sinuous road of isolating known or waiting to be discovered PhGs and iridoids.

Keywords Iridoids - Phenylethanoid glycosides · Centrifugal partition chromatography . Countercurrent chromatography $\cdot$ Counter-current chromatography $\cdot$ Natural products $\cdot$ Purification 


\section{Introduction}

Countercurrent separation (CCS) is widely used for the purification or analysis of bioactive molecules. It is a support-free liquid-liquid extraction technique, where both stationary and mobile phases are two immiscible liquids, with stationary phase being retained in the column due to gravitational and centrifugal forces (Ito 2005). CCS can be divided into countercurrent chromatography (CCC) and centrifugal partition chromatography (CPC) (Friesen et al. 2015). CCC mainly involves machines with a variable gravity field produced by a double axis planetary motion and a seal-free arrangement of the column (generally tubing wrapped around bobbins) and socalled "flying leads", tubing connecting the column(s) with ancillary equipment. CPC employs a constant gravity field produced by a single axis of rotation, together with rotatory seals for supply of solvent. CPC separation takes place in cells or chambers which are engraved in cartridges or disks tightly bolted together (Marston and Hostettmann 2006).

A series of favorable characteristics such as high loading capacity, lack of irreversible adsorption, low solvent consumption and ability to handle crude extracts, make CCS a frequently used technique for natural product separations (Ito 2005). Additionally, there are other irrefutable advantages: scale-up can be easily achieved; analytical grade solvents can be used, ensuring thus a high flexibility; the costs of purification is lower, as lower grade and more environmentally friendly solvents can be used; maximum sample recovery is observed (which is especially important in bioactivity-guided separation) and bioactivity can be preserved as solvent systems could be designed for a particular target molecule. Available instruments nowadays are automated, relatively user-friendly and designed to work at low pressures and high flow rates (Friesen et al. 2015).

Selection of a solvent system is the most crucial step in any CCS method development in order to achieve an optimum separation based on different partitioning of compounds between two liquid phases. Most of the available solvent and solute combinations that form a biphasic or multiphasic solvent system may be used in a CCS instrument. The wide range of possible combinations allows creating mixtures with a wide range of polarity and selectivity (Friesen et al.
2015). Two or more solvents may be mixed in an infinite number of proportions. The most important is to find the proper solvent system that provides the appropriate partition coefficient (K) value(s) to deliver the target compounds into the K value "sweet spot" of optimal resolution, which has been defined as $0.4<\mathrm{K}<2.5$ in regular elution mode (Friesen and Pauli 2007). A smaller K value results in a loss of peak resolution, whilst a higher value produces excessive band broadening.

The experimental procedure normally includes the determination of the partition coefficient of the target solute and related impurities in pre-selected biphasic solvent systems with the shake flask method. The concentration of the compound in each upper and lower phase is commonly determined by gas chromatography (GC) or high-performance liquid chromatography (HPLC) analysis (Hopmann et al. 2012).

Phenylethanoid glycosides (PhGs) and iridoids are two vast groups of water soluble secondary metabolites widely distributed in plant kingdom. Despite the fact that they have different structures and biosynthetic pathways, their co-existence in several plant families is a well-known fact. PhGs are structurally characterized by a $\beta$-glucopyranose core unit to which a hydroxyphenethyl (C6-C2) moiety is attached through a glycosidic bond. Phenylpropanoid (C6C3) residues, acetyl groups and other monosaccharides (rhamnose, apiose, galactose, xylose, etc.) are frequently linked to the glucose center of the molecule (Jimenez and Riguera 1994; Alipieva et al. 2014). Since most PhGs contain caffeic acid as aromatic acid moiety, they are also termed sometimes as caffeic acid glycoside esters, phenylpropanoid glycosides or caffeoyl phenylethanoid glycosides (Calis 2002). These compounds are not specific to any plant organ, being isolated from roots, barks, leaves, aerial parts as well as from callus and suspension cultures. The majority of the PhGs reported to date were found in the Scrophulariaceae, Oleaceae, Plantaginaceae, Lamiaceae and Orobanchaceae families. For example, verbascoside, the most prevalent representative, has been identified in more than 200 plant species belonging to 23 families (Schaluer et al. 2004). The early steps of the biogenetic pathways of PhGs are known, but several key enzymes and their corresponding genes remain to be discovered. However, the hydroxyphenethyl and phenylpropanoid moieties of a typical PhG (such as verbascoside) are synthetized 
from different types of precursors: tyrosine, tyramine and/or dopamine precursors by the shikimate pathway and phenylalanine via a cinnamate pathway. Tyramine is incorporated into PhGs through oxidation to the corresponding aldehyde, reduction to alcohol and, finally, $\beta$-glycosylation (Alipieva et al. 2014). In the case of dihydroxy derivatives, it was observed that the dihydroxy precursors (dopamine/L-DOPA) are less efficiently incorporated than their monohydroxy equivalents, suggesting that decarboxylation and deamination precede the hydroxylation at C-3 of the aromatic residues (Jimenez and Riguera 1994). Based on the number of sugar moieties, PhGs can be classified into monoglycosides (e.g. calcedariosides A, B, salidroside, plantainosides C and D), diglycosides (verbascoside, isoverbascoside, martynoside, forsythosides A and B) and triglycosides (alyssonoside, angoroside $\mathrm{C}$, cistanoside $\mathrm{A}$, echinacoside, wiedemannioside $\mathrm{C}$, leucosceptoside $\mathrm{B}$ ). PhGs have been increasingly brought to researchers' attention in the past few decades, as they were found to possess promising antioxidant, neuroprotective, antitumor, antiproliferative, hepatoprotective, antimicrobial, antiprotozoal, anti-inflammatory, immunomodulating and cardioprotective properties (Fu et al. 2008; Xue and Yang 2016). Some PhGs (verbascoside, oraposide, jionoside C) have already been included in several clinical trials (Jimenez and Riguera 1994). "General" verbascoside from Rehmaniae leaves (which is a mixture of PhGs extracted from Rehmania glutinosa Libosch.) reduced proteinuria and erythrocyturia in patients diagnosed with primary chronic glomerulonephritis after 8 weeks of administration (200 mg tablets, twice/day) alone or in combination with irbesartan (Qiu et al. 2013, 2014).

Iridoids form a perpetually expanding group of cyclopentano $[c]$ pyran monoterpenoids. Before the 1990s, there were 827 iridoid glycosides, secoiridoids, iridoid aglycones, iridoid derivatives and bisiridoids reported in literature (El-Naggar and Beal 1980; Boros and Stermitz 1990, 1991) and further 877 new iridoids were isolated from 1994 to 2010 (Dinda et al. 2007a, b, 2009, 2011). The bicyclic H-5/H-9 $\beta \beta$-cisfused cyclopentano[c]pyran ring system is the most common structural feature of these compounds; cleavage of cyclopentane ring produces secoiridoids, while cleavage of pyran ring produces cyclopentane derivatives (Dinda et al. 2007a). It was initially believed that iridoids are biogenetically derived from geranyl pyrophosphate through the action of terpene synthase, which generates cationic species that are subsequently cyclized and rearranged in hundreds of possible structures (Sampaio-Santos and Kaplan 2001). Later, when iridoid synthase was discovered by Geu-Flores et al. (2012), it was shown that its substrate is represented by 8-oxogeranial which is reduced to an enol intermediate using nicotinamide adenine dinucleotide phosphate (NADPH) that is further cyclized via a step-wise Michael addition to form the characteristic bicyclic 5-6 ring iridoid framework of nepetalactol; nepetalactol can easily give rise to any known iridoid (Lindner et al. 2014). The last steps in the biosynthesis of iridoids are considered to be $O$-glycosylation and $O$-alkylation. Iridoid synthesis is often regarded as an alternative route to the typical monoterpene biosynthesis. For instance, in Lamiaceae family, an inverse relationship between the production of monoterpenes and iridoids was observed (Sampaio-Santos and Kaplan 2001).

El-Naggar and Beal (1980) divided iridoids into several groups: iridoid glycosides with C-8, C-9 (e.g. catalpol, catalposide, harpagoside) and C-10 (e.g. gardenoside, geniposide, loganin) carbon skeleton, secoiridoids (e.g. gentiopicroside, morronoside, oleuropein, sweroside, swertiamarin), bisiridoids, bis-secoiridoids and non-glycosidic derivatives. Iridoids are present in numerous medicinal plants endowed with bitter, sedative, antipyretic, antitussive, wound healing and hypotensive properties. This fact encouraged the investigation of their bioactivities, leading to documented neuroprotective, anti-inflammatory, immunomodulating, hepatoprotective, cardioprotective, antitumor, antioxidant, antimicrobial, hypoglycemic, hypolipidemic, choleretic and spasmolytic effects (Tundis et al. 2008; Viljoen et al. 2012).

The conventional purification of PhGs and iridoids by semi-preparative HPLC and other types of column chromatography is time consuming, uses large amounts of organic solvents and requires repeated steps. Moreover, the sample recovery is low, because the hydroxyl groups of PhGs and iridoids make them strongly adsorbed onto the solid support (silica gel, Sephadex LH-20) during separation (Li et al. 2005). Therefore, CCS comes as an alternative tool to overcome the issues associated with solid-phase adsorbents and it has been successfully used for the separation of various natural products (Marston and Hostettmann 2006). 


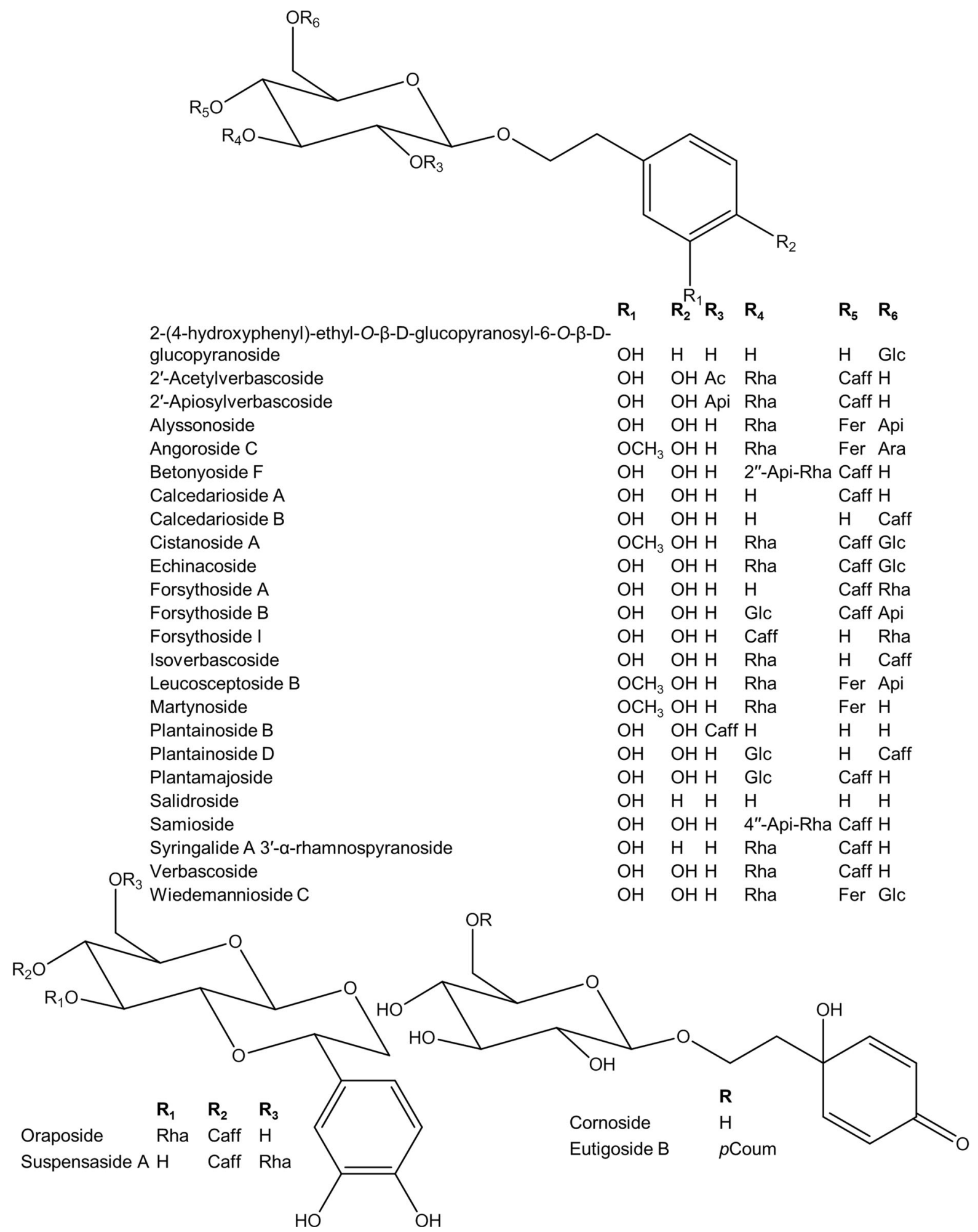

Fig. 1 Phenylethanoid glycosides isolated through countercurrent separation. Ac acetyl, Api apiosyl, Ara arabinosyl, Caff caffeoyl, Fer feruloyl, Glc glucosyl, pCoum p-coumaroyl, Rha rhamnosyl 


\section{Methodology}

In order to assess the use of two phase solvent systems in isolation of PhGs and iridoids, the search for papers describing all aspects concerning their CCS was carefully performed in online databases (Scopus, PubMed). In the first step, all possible terms denoting the technique ("counter-current chromatography", "countercurrent chromatography", "CCC", "HSCCC", "HPCCC", "centrifugal partition chromatography", "CPC") were paired one by one with terms designating the class of interest compounds ("iridoid", "bisiridoid", "secoiridoid", "phenylethanoid glycosides", "phenylpropanoid glycosides"). However, when the search was performed with the latter combination, some of the accessed results were not taken into consideration as not all phenylpropanoid glycosides are structurally included in the phytochemical class of PhGs (syringine, eleutheroside D). Next, each listed compound was searched in the above mentioned databases in combination with previously described terminologies related to CCS techniques. Some compounds, especially phenylethanoid mono- and di-glycosides, raised confusions regarding their nomenclature. For example, verbascoside, acteoside, kusaginin and orabanchin have the same chemical structure of 2-(3,4-dihydroxyphenyl)ethyl-1-O- $\alpha$-L-rhamnopyranosyl- $(1 \rightarrow 3)-(4-O-E-$

caffeoyl)- $\beta$-D-glucopyranoside. Other examples are isoverbascoside often named as isoacteoside, oraposide as orabanchoside or crenatoside, and calcedarioside often referred to as calceolarioside. Thus, when the database searches were conducted, all designations were used as keywords, but in order to avoid confusion, only "verbascoside", "isoverbascoside", "oraposide" and "calcedarioside" were subsequently employed as terminology in this paper.

Finally, the authors collected 65 research papers from the last 20 years (1998-2018) describing CCC and CPC isolation of PhGs and iridoids. In total, 84 different phytochemicals (Figs. 1,2) were separated from 42 plant species belonging to 16 distinct families. The results are presented hereafter in form of two tables (Tables 1,2) that sum up the most important features of the CCS of PhGs and iridoids. Undoubtedly, the information about the source of the target compounds (plant name, family, plant parts) is primarily given. As extraction is the first step that dictates the type and complexity of a matrix, the extraction solvent is further specified. Since yield and purity are important for method development, data about the sample loading, yield and purity of isolated compounds were provided. Of course, the operating parameters for CCS, such as type of technique (CCC/ $\mathrm{CPC}$ ), elution mode (isocratic/step-gradient/step-flow gradient, dual mode, NP, RP) as well as the composition of the solvent systems and $\mathrm{K}$ values were also given in these tables. Moreover, in order to give the readers an idea about the number of isolation steps required, up- and down-stream complementary chromatographic techniques (column chromatography, thin-layer chromatography, semi-preparative HPLC) were included. Further insights are provided in the Electronic Supplementary Material. The data in the Excel table are arranged in alphabetical order of the plant species, with additional subclassification of each compound, sample loading details (sample loading per injection, sample volume, sample concentration, column volume, sample loaded per each $100 \mathrm{~mL}$ of column volume, sample solvent, and sample solvent ratio), CCC/CPC column details (internal diameters or number of cells), operating parameters (column's revolution speed, mobile phase flow-rate, eluant detection wavelength), instrument model and manufacturer. One of the main differences as compared to Tables 1 and 2 comes from the fact that normalized solvent ratios were given in this file, in order to make solvent systems easily comparable among each other. Another aspect that was taken into consideration regarding the organization of this file was that compounds within the same plant species were placed according to their elution order from the CCS column. Moreover, the $\log \mathrm{P}$ values, a parameter important for estimating the distribution of a compound between two immiscible phases, were calculated using ChemDraw Ultra 12.0 software. As we are not aware of the existence of a similar work, this study can act as a starting point for CCS users that target the isolation of compounds belonging to these two phytochemical classes. In the next section, an extensive discussion on the use of CCS in isolating PhGs and iridoids with focus on employed solvent systems is presented. Finally, the last part is devoted to analyzing the biological relevance of $\mathrm{PhGs}$ and iridoids isolated through CCS. 
<smiles>[R]CC1([R6])C([R3])C([R4])C2([R3])C([R2])=COC([R1])C12</smiles><smiles>OCC1=CC(O)C2C=COC(Cl)C12</smiles>

$R_{1}$

6- $\beta$-hydroxyantirrhide

7-O-(4-maloyl)loganin

7-Dehydrologanin

8-O-acetylharpagide

10-Hydroxyhastatoside

10-Hydroxycornin

Caprarioside

Chlorotuberside

Harpagoside

Ipolamiide

Loganetin

Loganic acid

Loganin

Penstemonoside

Phloyoside II

Shanzhiside methyl ester Glc<smiles>[R2]C(=O)C1=COC([O-])C2C1C([R2])=CC2([R3])CO</smiles>

$\begin{array}{lll}\mathbf{R}_{\mathbf{1}} & \mathbf{R}_{\mathbf{2}} & \mathbf{R}_{\mathbf{3}}\end{array}$

6- $\beta$-Hydroxy geniposide $\mathrm{CH}_{3} \mathrm{OH} \mathrm{H}$

Gardenoside

Geniposide

Geniposidic acid
$\mathrm{CH}_{3} \mathrm{H} \quad \mathrm{OH}$

$\mathrm{CH}_{3} \mathrm{H} \quad \mathrm{H}$

$\mathrm{H} \mathrm{H} \quad \mathrm{H}$ $\mathrm{H} \mathrm{OHOH}=\mathrm{CH}_{2}$
$\mathbf{R}_{\mathbf{2}}$

$\begin{array}{lllll}\mathbf{R}_{\mathbf{3}} & \mathbf{R}_{\mathbf{4}} & \mathbf{R}_{\mathbf{5}} & \mathbf{R}_{\mathbf{6}} & \mathbf{R}_{\mathbf{7}}\end{array}$ $\mathrm{COOCH}_{3} \mathrm{H} \quad \mathrm{H}$ OMal H H $\mathrm{COOCH}_{3} \mathrm{H} \mathrm{H}=\mathrm{O} \quad \mathrm{H} \quad \mathrm{H}$ $\mathrm{H} \quad \mathrm{OHOHH}$ OAc $\mathrm{H}$ $\mathrm{COOCH}_{3} \mathrm{OH}=\mathrm{OH} \quad \mathrm{H} \quad \mathrm{OH}$ $\mathrm{COOCH}_{3} \mathrm{H}=\mathrm{OH} \quad \mathrm{H} \quad \mathrm{OH}$ $\mathrm{H} \quad \mathrm{OHOHH} \mathrm{OBz} \mathrm{H}$ $\mathrm{COOCH}_{3} \mathrm{H} \mathrm{OHCl} \mathrm{OH} \mathrm{H}$ $\mathrm{H} \quad \mathrm{OHOHH}$ OCinn $\mathrm{H}$ $\mathrm{COOCH}_{3} \mathrm{OHH} \quad \mathrm{H} \quad \mathrm{OH} \quad \mathrm{H}$ $\mathrm{COOCH}_{3} \mathrm{H} \mathrm{H} \mathrm{OH} \mathrm{H} \mathrm{H}$ $\mathrm{COOH} \mathrm{H} \mathrm{H} \mathrm{OH} \mathrm{H} \quad \mathrm{H}$ $\mathrm{COOCH}_{3} \mathrm{H}$ H OH H H $\mathrm{COOCH}_{3} \mathrm{OHOHH} \quad \mathrm{H} \quad \mathrm{H}$ $\mathrm{COOCH}_{3} \mathrm{OHOHCl} \mathrm{OH} \mathrm{H}$ $\mathrm{COOCH}_{3} \mathrm{H} \mathrm{OHH} \quad \mathrm{OH} H$<smiles>[R20]C1C2OC2(C)C2C([O-])OC=CC12[R]</smiles><smiles>OC1CC2(O)C(O)C(Cl)C3(O)COC(O1)C23</smiles>

3-Hydroxymyopochlorin<smiles>OC1C(Cl)C2(O)C(O)OC3CC1(O)C2O3</smiles>

5-Hydroxyglutinoside<smiles>COC1=CCC2C(COC3OC(CO)CCC3O)=COC(OC(=O)CC(C)C)C12</smiles><smiles>CC(=O)C1=COC2OC1CC2C(C)=O</smiles>

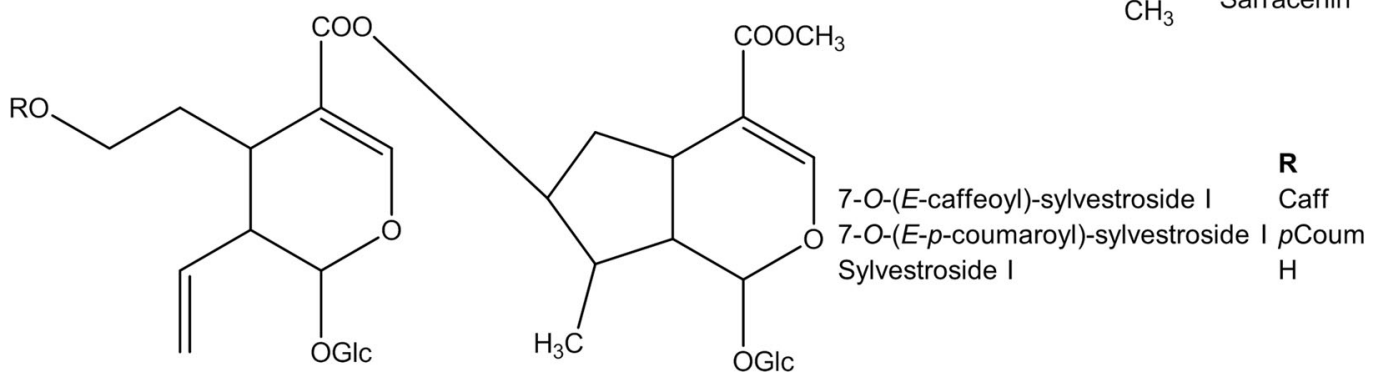


४Fig. 2 Iridoids isolated through countercurrent separation. $A c$ acetyl, Bz benzyl, Caff caffeoyl, Cinn cinnamoyl, Fer feruloyl, Glc glucosyl, Mal maloyl, $p$ Coum $p$-coumaroyl, Van vanilloyl

CCS application to phenylethanoid glycosides and iridoids purification

A total of 28 distinct $\mathrm{PhGs}$ and 56 iridoids were isolated using 59 different biphasic solvent systems. Since PhGs and iridoids are highly polar molecules, $66 \%$ of all employed solvent systems contained a minimum of $45 \%$ water. About $56 \%$ of the solvent systems were composed of various ternary ratios of ethyl acetate-alcohol-water or binary systems, such as ethyl acetate-water and $n$-butanol-water. Chlorinated solvents (chloroform, dichloromethane) were present in $18 \%$ of the solvent systems and mainly employed for iridoids separations using CCC only. One of these solvent systems contained acetic acid as $\mathrm{pH}$ modifier. Chlorinated solvents provide very good solubility for natural products extracts but are mostly avoided nowadays for safety restrictions. Three alkane based systems $(5 \%)$ employed for purification of secoiridoids and C-9 iridoids included combinations, such as $n$-hexane-ethyl acetate- $n$-butanol-water, $n$-hexane$n$-butanol-water and $n$-hexane- $n$-butanol-methanolwater-acetic acid. These systems were mostly exceptions for this polarity level of target compounds. From another point of view, the solvent systems used to separate PhGs and iridoids were most often formulated with three $(65 \%)$ or four $(31 \%)$ solvent combinations using short-chain alcohols (methanol, ethanol, 1- or 2-propanol) as modifiers to reduce the polarity difference between the two liquid phases.

CCC [also referred as high-speed CCC (HSCCC) or high-performance CCC (HPCCC)] was by far the most used technique, being reported in $83 \%$ of the papers, followed by centrifugal partition chromatography (CPC). This can be simply explained by a wider distribution of CCC instruments across the international research community, especially in China, and their lower cost and ease of manufacture. There is almost equal number of CPC and CCC instruments used in industry these days but access to their applications is rather limited (Ignatova and Sutherland 2015). Out of 22 common solvent systems for PhGs separations with CCC, $64 \%$ were ethyl acetate $-n$ butanol-water family with ratios depending on anatomical part of a plant (Fig. 3). $73 \%$ of CPC papers were about iridoids purifications with ethyl acetate- $n$-propanol-water (35-30:15-20:50) as the most employed solvent systems. The content of ethyl acetate and $n$-butanol varied in moving from iridoids C-9 to C-10, followed by secoiridoids and bissecoiridoids, according to their decreasing polarity order. The least polar system $t$-butyl methyl etheracetonitrile-water (30:30:40) was used for the isolation of non-glycosidic iridoids. Some variations in the mode of operation of the CCC and CPC columns were observed during the survey, including step gradient elution (de Juliao et al. 2010; Leitao et al. 2015), enhancement of flow rate (Liang et al. 2013b; Rho et al. 2016), two-step CCS (Lei et al. 2001b; Lemus et al. 2015; Li et al. 2008), elution-extrusion CCS (Chen et al. 2011; Liang et al. 2018) and recycling CCS (Chen et al. 2011).

\section{CCS and phenylethanoid glycosides' isolation}

The $28 \mathrm{PhGs}$ isolated through CCS belonged to eight different plant families: Verbenaceae, Orobonchaceae, Scrophulariaceae, Plantaginaceae, Oleaceae, Lamiaceae, Crassulaceae and Gesneriaceae. Verbascoside and isoverbascoside were by far the most frequently isolated PhGs (23 and 8 times, respectively), either intentionally or as part of a larger group (Table 1). Possessing a wider polarity range as compared to monoglycosides and triglycosides, phenylethanoid diglycosides are the most distributed class of PhGs. Consequently, a high number of CCS applications focused on their isolations. Nevertheless, they were also separated altogether with mono-/triglycosides from various plant matrices $[$ mono + di: Abeliophyllum distichum (Li et al. 2013), Chirita longgangensis (Duan et al. 2014); di + tri: Cistanche deserticola (Li et al. 2008); Lamiophlomis rotata (Yue et al. 2013a), Lantana trifolia (de Juliao et al. 2010), Pedicularis longiflora var. tubiformis (Zhang et al. 2015), Penstemon barbatus (Wang et al. 2013)].

Out of the 21 plant species, PhGs were mainly isolated by CCC from extracts of roots (8), stems (4), whole plants (5) and upper parts [aerial parts (3), leaves (5)], with 14 solvent systems based on ethyl acetate- $n$-butanol-water family (Fig. 3 ). The matrix of a plant extract and its complexity (including additional purification steps before CCS) has obvious effects on the solvent system polarity. For the 
<smiles>[X]C1=C2C(=O)OCC=C2C(=O)C2=COC([R2])C([R3])C2([R3])CC([R])([R2])OC1C(=O)OCCc1ccc([R])c(O)c1</smiles>

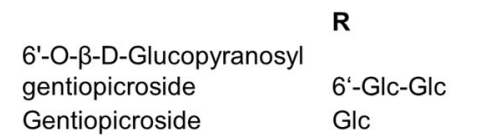<smiles>[R]CC1C(C(C)=O)=COC(OC(=O)[O-])C1C=C</smiles><smiles>[R]C(O)C(=O)OC(=O)OC(=O)O</smiles>
Secoxyloganin $\mathrm{COOH}$
$\begin{array}{llllll}X & \mathbf{R}_{\mathbf{1}} & \mathbf{R}_{\mathbf{2}} & \mathbf{R}_{\mathbf{3}} & \mathbf{R}_{\mathbf{4}}\end{array}$ 8-Hydroxy-10-hydrosweroside $\mathrm{CH}_{2} \mathrm{H} \quad \mathrm{H} \quad \mathrm{H} \quad \mathrm{CH}(\mathrm{OH})-\mathrm{CH}_{3}$ Eustomoruside $\quad \mathrm{O} \quad \mathrm{H} \quad \mathrm{H} \quad \mathrm{OH} \mathrm{CH}(\mathrm{OH})-\mathrm{CH}_{2}-\mathrm{CH}_{3}$ Eustomoside Isovogeloside Sweroside Swertiamarin Vogeloside
$\mathrm{O} \mathrm{H} \quad \mathrm{H} \quad \mathrm{OH}$

$\mathrm{O} \mathrm{OCH}_{3} \mathrm{H} \quad \mathrm{H} \quad \mathrm{CH}=\mathrm{CH}_{2}$

$\mathrm{H} \quad \mathrm{H} \quad \mathrm{H} \quad \mathrm{CH}=\mathrm{CH}_{2}$

$\mathrm{O} \quad \mathrm{H} \quad \mathrm{OH} \mathrm{CH}=\mathrm{CH}_{2}$

$\mathrm{O} \quad \mathrm{H} \quad \mathrm{OCH}_{3} \mathrm{H} \quad \mathrm{CH}=\mathrm{CH}_{2}$

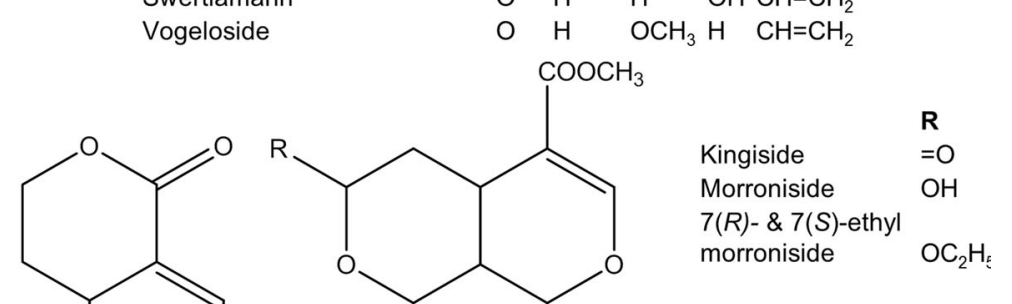
$\mathrm{HO}$

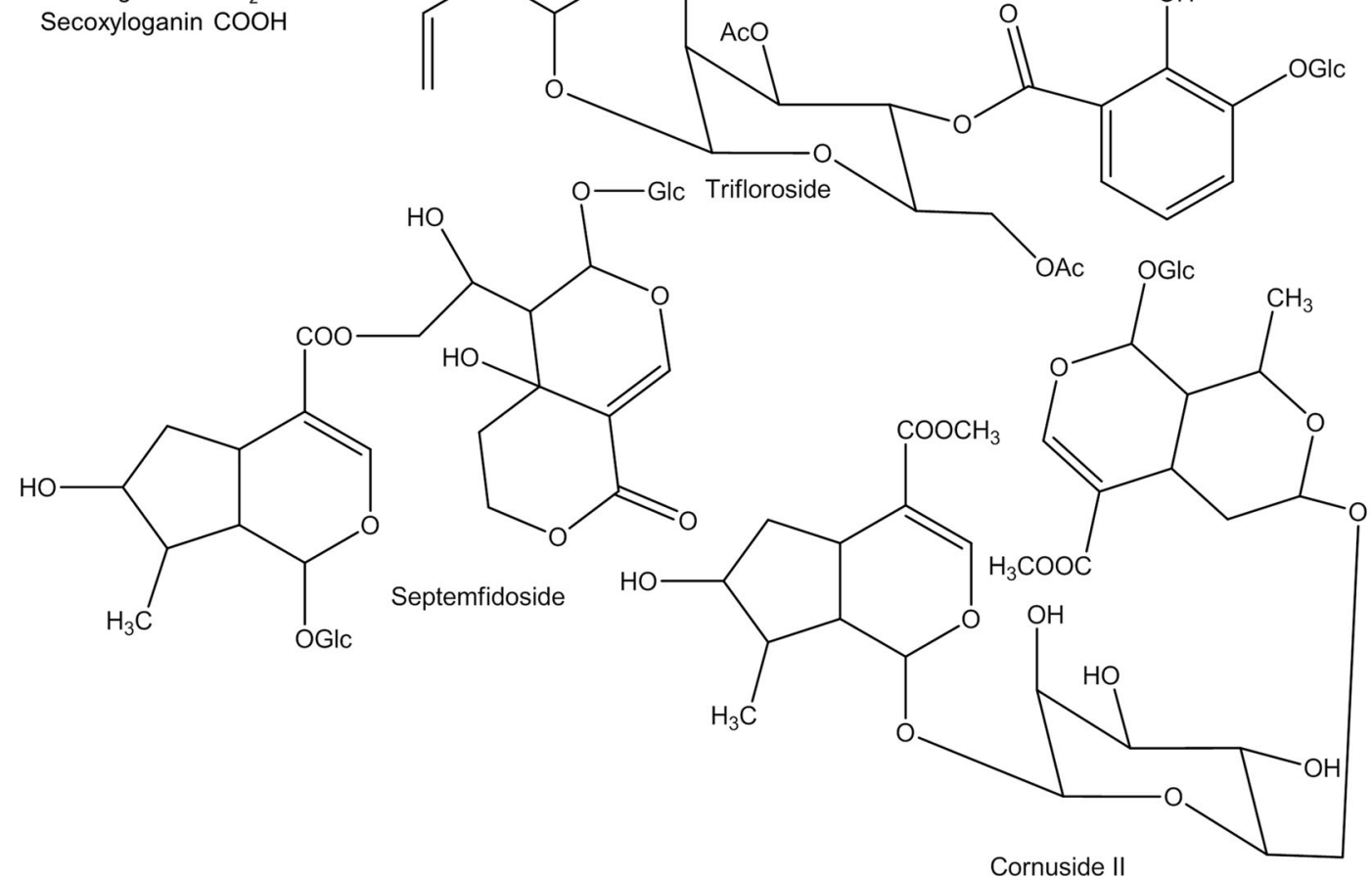<smiles>CC(COC(=O)OC(C)C)CC(C)OC(C)C</smiles>

$7(R)$ - \& 7(S)-ethyl morroniside $\quad \mathrm{OC}_{2} \mathrm{H}_{?}$

Fig. 2 continued 
Table 1 List of phenylethanoid glycosides isolated through countercurrent separation

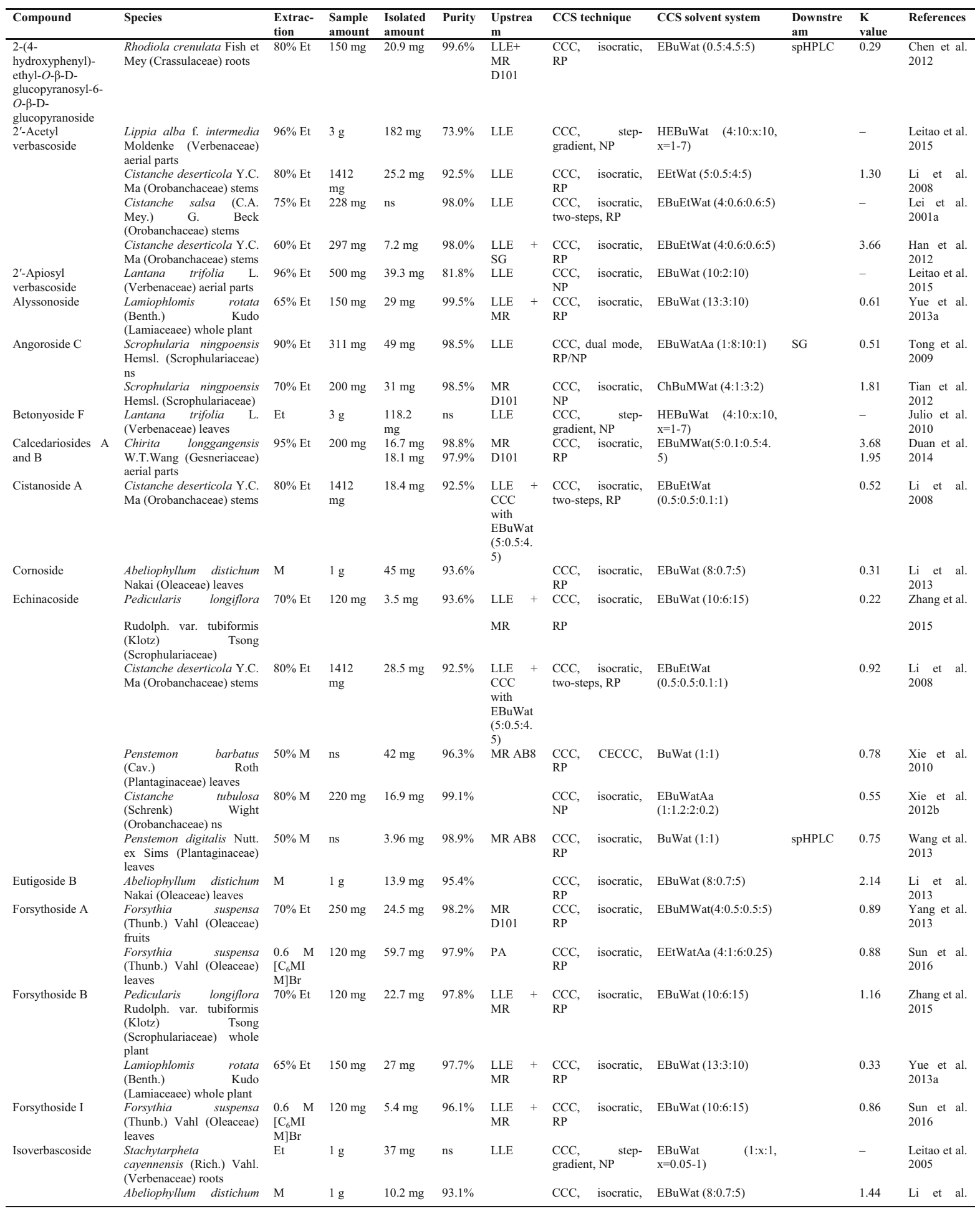


Table 1 continued

\begin{tabular}{|c|c|c|c|c|c|c|c|c|c|c|c|}
\hline Compound & Species & $\begin{array}{l}\text { Extrac- } \\
\text { tion }\end{array}$ & $\begin{array}{l}\text { Sample } \\
\text { amount }\end{array}$ & $\begin{array}{l}\text { Isolated } \\
\text { amount }\end{array}$ & Purity & $\begin{array}{l}\text { Upstrea } \\
\text { m }\end{array}$ & CCS technique & CCS solvent system & $\begin{array}{l}\text { Downstre } \\
\text { am }\end{array}$ & $\begin{array}{l}\mathbf{K} \\
\text { value }\end{array}$ & References \\
\hline & Nakai (Oleaceae) leaves & & & & & & RP & & & & 2013 \\
\hline & $\begin{array}{lr}\text { Pedicularis } & \text { longiflora } \\
\text { Rudolph. var. } & \text { tubiformis } \\
\text { (Klotz) } & \text { Tsong } \\
\text { (Scrophulariaceae) } & \text { whole } \\
\text { plant } & \end{array}$ & $70 \% \mathrm{Et}$ & $40 \mathrm{mg}$ & $18 \mathrm{mg}$ & $98.0 \%$ & $\begin{array}{l}\text { LLE }+ \\
\text { SG }\end{array}$ & $\begin{array}{l}\mathrm{CCC} \text {, isocratic, } \\
\mathrm{RP}\end{array}$ & ChBuMWat $(4: 3: 4: 5)$ & & 0.83 & $\begin{array}{l}\text { Chen et al. } \\
2014\end{array}$ \\
\hline & $\begin{array}{l}\text { Cistanche deserticola Y.C. } \\
\text { Ma (Orobanchaceae) ns }\end{array}$ & $80 \% \mathrm{Et}$ & $\begin{array}{l}1412 \\
\mathrm{mg}\end{array}$ & $30.1 \mathrm{mg}$ & $92.5 \%$ & LLE & $\begin{array}{l}\text { CCC, isocratic, } \\
\text { two-steps, RP }\end{array}$ & EEtWat $(5: 0.5: 4.5)$ & & 1.11 & $\begin{array}{l}\mathrm{Li} \text { et al. } \\
2008\end{array}$ \\
\hline & $\begin{array}{l}\text { Plantago psyllium L. } \\
\text { (Plantaginaceae) seeds }\end{array}$ & $80 \% \mathrm{M}$ & $978 \mathrm{mg}$ & $17.5 \mathrm{mg}$ & $94.0 \%$ & LLE & $\begin{array}{l}\text { CCC, isocratic, } \\
\text { RP }\end{array}$ & EWat $(1: 1)$ & & 0.92 & $\mathrm{Li}_{2005}$ et al. \\
\hline & $\begin{array}{lr}\text { Cistanche } & \text { tubulosa } \\
\text { (Schrenk) } & \text { Wight } \\
\text { (Orobanchaceae) } & \text { stems }\end{array}$ & $\mathrm{M}^{*}$ & $214 \mathrm{mg}$ & $\mathrm{ns}$ & $95 \%$ & $\begin{array}{l}\text { Sephade } \\
\text { x LH-20 }\end{array}$ & $\begin{array}{l}\text { CCC, isocratic, } \\
\text { two-steps, RP }\end{array}$ & $\begin{array}{l}\text { EBuEtWat }(35: 6: 6: 50 \\
\text { and } 30: 10: 6: 50)\end{array}$ & & - & $\begin{array}{l}\text { Lei et al. } \\
2001 b\end{array}$ \\
\hline & $\begin{array}{l}\text { Cistanche deserticola Y.C. } \\
\text { Ma (Orobanchaceae) stems }\end{array}$ & $60 \% \mathrm{Et}$ & $297 \mathrm{mg}$ & $13 \mathrm{mg}$ & $95.0 \%$ & LLE & $\begin{array}{l}\mathrm{CCC} \text {, isocratic, } \\
\mathrm{RP}\end{array}$ & EBuEtWat (4:0.6:0.6:5) & & 1.29 & $\begin{array}{l}\text { Han et al. } \\
2012\end{array}$ \\
\hline Leucosceptoside B & $\begin{array}{lr}\text { Lamiophlomis } & \text { rotata } \\
\text { (Benth.) } & \text { Kudo }\end{array}$ & $65 \% \mathrm{Et}$ & $150 \mathrm{mg}$ & $13 \mathrm{mg}$ & $97.3 \%$ & LLE & $\begin{array}{l}\mathrm{CCC} \text {, isocratic, } \\
\mathrm{RP}\end{array}$ & EBuWat $(13: 3: 10)$ & & 1.19 & $\begin{array}{l}\text { Yue et al. } \\
2013 \mathrm{a}\end{array}$ \\
\hline Martynoside & $\begin{array}{l}\text { (Lamiaceaee) whole plant } \\
\text { Stachytarpheta } \\
\text { cayennensis (Rich.) Vahl. } \\
\text { (Verbenaceae) roots }\end{array}$ & Et & $1 \mathrm{~g}$ & $55 \mathrm{mg}$ & ns & LLE & $\begin{array}{l}\mathrm{CCC}, \quad \text { step- } \\
\text { gradient, NP }\end{array}$ & $\begin{array}{l}\text { EBuWat } \quad(1: \mathrm{x}: 1, \\
\mathrm{x}=0.05-1)\end{array}$ & & - & $\begin{array}{l}\text { Leitao et al. } \\
2005\end{array}$ \\
\hline & $\begin{array}{l}\text { Lantana trifolia } \\
\text { (Verbenaceae) leaves }\end{array}$ & Et & $3 \mathrm{~g}$ & $5.8 \mathrm{mg}$ & ns & LLE & $\begin{array}{l}\mathrm{CCC}, \quad \text { step- } \\
\text { gradient, NP }\end{array}$ & $\begin{array}{l}\text { HEBuWat } \quad(4: 10: 1: 10 \text {, } \\
\mathrm{x}=1-7)\end{array}$ & $\begin{array}{l}\text { Sephadex } \\
\text { LH-20 }\end{array}$ & - & $\begin{array}{l}\text { Juliao et al. } \\
2010\end{array}$ \\
\hline Oraposide & $\begin{array}{ll}\text { Orobanche } & \text { rapum- } \\
\text { genistae } & \text { Thuill. } \\
\text { (Orobanchaceae) } & \text { whole } \\
\text { plant } & \end{array}$ & $70 \% \mathrm{Et}$ & $300 \mathrm{mg}$ & $30 \mathrm{mg}$ & $82 \%$ & & $\begin{array}{l}\mathrm{CCC} \text {, isocratic, } \\
\mathrm{RP} / \mathrm{NP}\end{array}$ & $\begin{array}{l}\text { EAcWat } \\
\text { RP: } 2.6: 2.6: 4.8 \\
\text { NP: }+2 \% \mathrm{M}\end{array}$ & & 1.15 & $\begin{array}{l}\text { Viron et al. } \\
1998\end{array}$ \\
\hline & $\begin{array}{l}\text { Orobanche rapum genistae } \\
\text { Thuill. (Orobanchaceae) } \\
\text { whole plant }\end{array}$ & $70 \% \mathrm{Et}$ & $6 \mathrm{~g}$ & $450 \mathrm{mg}$ & $100 \%$ & & $\begin{array}{l}\mathrm{CPC} \text {, isocratic, } \\
\mathrm{RP}\end{array}$ & EAtWat (10.6:1:9.7) & & - & $\begin{array}{l}\text { Viron et al. } \\
2000\end{array}$ \\
\hline $\begin{array}{l}\text { Plantainosides } \\
\text { and D }\end{array}$ & $\begin{array}{l}\text { Chirita longgangensis } \\
\text { W.T.Wang (Gesneriaceae) } \\
\text { aerial parts }\end{array}$ & $95 \% \mathrm{Et}$ & $200 \mathrm{mg}$ & $\begin{array}{l}9.5 \mathrm{mg} \\
28.4 \mathrm{mg}\end{array}$ & $\begin{array}{l}94.4 \% \\
97.6 \%\end{array}$ & $\begin{array}{l}\text { MR } \\
\text { D101 }\end{array}$ & $\begin{array}{l}\mathrm{CCC} \text {, isocratic, } \\
\mathrm{RP}\end{array}$ & $\begin{array}{l}\text { EBuMWat(5:0.1:0.5:4. } \\
5)\end{array}$ & & $\begin{array}{l}0.82 \\
0.35\end{array}$ & $\begin{array}{l}\text { Duan et al. } \\
2014\end{array}$ \\
\hline Plantamajoside & $\begin{array}{l}\text { Plantago asiatica L. } \\
\text { (Plantaginaceae) aerial } \\
\text { parts }\end{array}$ & $80 \% \mathrm{Et}$ & $1.341 \mathrm{~g}$ & $45.6 \mathrm{mg}$ & $93.3 \%$ & LLE & $\begin{array}{l}\text { CPC, isocratic, } \\
\text { RP }\end{array}$ & $\begin{array}{l}\text { EBuEtWat } \\
(0.5: 0.5: 0.1: 1)\end{array}$ & & 2.69 & $\begin{array}{l}\mathrm{Li} \text { et al. } \\
2009\end{array}$ \\
\hline Salidroside & $\begin{array}{l}\text { Rhodiola crenulata Fish et } \\
\text { Mey (Crassulaceae) roots }\end{array}$ & $\begin{array}{l}\text { AtWat } \\
(70: 30)\end{array}$ & $\begin{array}{l}1216 \\
\mathrm{mg}\end{array}$ & $21.9 \mathrm{mg}$ & $98.0 \%$ & LLE & $\begin{array}{l}\text { CCC, iscratic, } \\
\text { two-steps, RP }\end{array}$ & $\begin{array}{l}\text { EBuWat }(1: 4: 5) \text { and } \\
\text { ChMIsoWat }(5: 6: 1: 4)\end{array}$ & & - & $\begin{array}{l}\text { Han et al. } \\
2002\end{array}$ \\
\hline & $\begin{array}{l}\text { Rhodiola sachalinensis A. } \\
\text { Bor (Crassulaceae) roots }\end{array}$ & M & $250 \mathrm{mg}$ & $32 \mathrm{mg}$ & $98.0 \%$ & & $\begin{array}{l}\mathrm{CCC} \text {, isocratic, } \\
\mathrm{RP}\end{array}$ & EBuWat $(3: 2: 5)$ & & 0.52 & $\begin{array}{l}\text { Li and } \\
\text { Chen } 2001\end{array}$ \\
\hline & $\begin{array}{ll}\text { Rhodiola rosea } & \mathrm{L} . \\
\text { (Crassulaceae) roots } & \end{array}$ & M & $100 \mathrm{mg}$ & $0.5 \mathrm{mg}$ & $90.0 \%$ & LLE & $\begin{array}{l}\mathrm{CCC}, \text { isocratic, } \\
\mathrm{RP}\end{array}$ & EBuWat $(3: 2: 5)$ & & 0.65 & $\begin{array}{l}\text { Mudge et } \\
\text { al. } 2012\end{array}$ \\
\hline & $\begin{array}{l}\text { Rhodiola crenulata Fish et } \\
\text { Mey (Crassulaceae) roots }\end{array}$ & $80 \% \mathrm{Et}$ & $150 \mathrm{mg}$ & $\begin{array}{l}120.2 \\
\mathrm{mg}\end{array}$ & $99.1 \%$ & LLE & $\begin{array}{l}\mathrm{CCC}, \text { isocratic, } \\
\mathrm{RP}\end{array}$ & EBuWat $(0.5: 4.5: 5)$ & spHPLC & 0.85 & $\begin{array}{l}\text { Chen et al. } \\
2012\end{array}$ \\
\hline Samioside & $\begin{array}{l}\text { Lantana trifolia } \\
\text { (Verbenaceae) leaves }\end{array}$ & Et & $3 \mathrm{~g}$ & $5.9 \mathrm{mg}$ & ns & LLE & $\begin{array}{l}\mathrm{CCC}, \quad \text { step- } \\
\text { gradient, NP }\end{array}$ & $\begin{array}{l}\text { HEBuWat } \quad(4: 10: 1: 10 \\
\mathrm{x}=1-7)\end{array}$ & $\begin{array}{l}\text { Sephadex } \\
\text { LH-20 } \\
\text { and } \\
\text { spHPLC }\end{array}$ & - & $\begin{array}{l}\text { Juliao et al. } \\
2010\end{array}$ \\
\hline Suspensaside A & $\begin{array}{l}\text { Forsythia suspensa } \\
\text { (Thunb.) Vahl (Oleaceae) } \\
\text { fruits }\end{array}$ & $70 \% \mathrm{Et}$ & $250 \mathrm{mg}$ & $8.8 \mathrm{mg}$ & $98.0 \%$ & $\begin{array}{l}\text { MR } \\
\text { D101 }\end{array}$ & $\begin{array}{l}\mathrm{CCC}, \text { isocratic, } \\
\mathrm{RP}\end{array}$ & EBuMWat (4:0.5:0.5:5) & & 1.94 & $\begin{array}{l}\text { Yang et al. } \\
2013\end{array}$ \\
\hline $\begin{array}{l}\text { Syringalide A } 3 \text { '- } \alpha- \\
\text { rhamnospyranoside }\end{array}$ & $\begin{array}{l}\text { Cistanche deserticola Y.C. } \\
\text { Ma (Orobanchaceae) stems }\end{array}$ & $60 \% \mathrm{Et}$ & $297 \mathrm{mg}$ & $12.5 \mathrm{mg}$ & $99.0 \%$ & LLE & $\begin{array}{l}\mathrm{CCC}, \text { isocratic, } \\
\mathrm{RP}\end{array}$ & EBuEtWat (4:0.6:0.6:5) & & 2.56 & $\begin{array}{l}\text { Han et al. } \\
2012\end{array}$ \\
\hline Verbascoside & $\begin{array}{l}\text { Stachytarpheta } \\
\text { cayennensis (Rich.) Vahl. } \\
\text { (Verbenaceae) roots }\end{array}$ & Et & $1 \mathrm{~g}$ & $300 \mathrm{mg}$ & ns & LLE & $\begin{array}{l}\mathrm{CCC}, \quad \text { step- } \\
\text { gradient, NP }\end{array}$ & $\begin{array}{l}\text { EBuWat } \quad(1: \mathrm{x}: 1, \\
\mathrm{x}=0.05-1)\end{array}$ & & - & $\begin{array}{l}\text { Leitao et al. } \\
2005\end{array}$ \\
\hline & $\begin{array}{l}\text { Abeliophyllum distichum } \\
\text { Nakai (Oleaceae) leaves }\end{array}$ & M & $1 \mathrm{~g}$ & $36.2 \mathrm{mg}$ & $94.0 \%$ & & $\begin{array}{l}\mathrm{CCC} \text {, isocratic, } \\
\mathrm{RP}\end{array}$ & EBuWat (8:0.7:5) & & 1.06 & $\begin{array}{l}\mathrm{Li} \text { et al. } \\
2013\end{array}$ \\
\hline & $\begin{array}{l}\text { Lippia alba f. intermedia } \\
\text { Moldenke (Verbenaceae) } \\
\text { aerial parts }\end{array}$ & $96 \% \mathrm{Et}$ & $3 \mathrm{~g}$ & $589 \mathrm{mg}$ & $57.9 \%$ & LLE & $\begin{array}{l}\mathrm{CCC}, \quad \text { step- } \\
\text { gradient, NP }\end{array}$ & $\begin{array}{l}\text { HEBuWat } \quad(4: 10: x: 10, \\
x=1-7)\end{array}$ & & - & $\begin{array}{l}\text { Leitao et al. } \\
2015\end{array}$ \\
\hline & $\begin{array}{l}\text { Lippia javanica Spreng. } \\
\text { (Verbenaceae) leaves }\end{array}$ & $50 \% \mathrm{M}$ & $\begin{array}{l}1247 \\
\mathrm{mg}\end{array}$ & ns & $71.0 \%$ & SG & $\begin{array}{l}\text { CCC, dual mode, } \\
\text { NP/RP }\end{array}$ & EBuWat $(10: 1: 10)$ & & - & $\begin{array}{l}\text { Oyourou et } \\
\text { al. } 2013\end{array}$ \\
\hline & $\begin{array}{l}\text { Lantana trifolia L. } \\
\text { (Verbenaceae) aerial parts }\end{array}$ & $96 \% \mathrm{Et}$ & $500 \mathrm{mg}$ & $29.5 \mathrm{mg}$ & $80.5 \%$ & LLE & $\begin{array}{l}\text { CCC, isocratic, } \\
\text { NP }\end{array}$ & EBuWat $(10: 2: 10)$ & & - & $\begin{array}{l}\text { Leitao et al. } \\
2015\end{array}$ \\
\hline & $\begin{array}{l}\text { Lantana trifolia L. } \\
\text { (Verbenaceae) leaves }\end{array}$ & Et & $3 \mathrm{~g}$ & $\begin{array}{l}418.5 \\
\mathrm{mg}\end{array}$ & ns & LLE & $\begin{array}{l}\text { CCC, two-steps, } \\
\text { step-gradient and } \\
\text { isocratic, RP }\end{array}$ & $\begin{array}{l}\text { HEBuWat }(4: 10: x: 10, \\
x=1-7) \text { and HEBuWat } \\
(4: 10: 6: 10)\end{array}$ & & - & $\begin{array}{l}\text { Juliao et al. } \\
2010\end{array}$ \\
\hline & $\begin{array}{lr}\text { Pedicularis } & \text { longiflora } \\
\text { Rudolph. var. } & \text { tubiformis } \\
\text { (Klotz) } & \text { Tsong } \\
\text { (Scrophulariaceae) } & \text { whole } \\
\text { plant } & \end{array}$ & $70 \% \mathrm{Et}$ & $40 \mathrm{mg}$ & $20 \mathrm{mg}$ & $97.0 \%$ & LLE & $\begin{array}{l}\mathrm{CCC} \text {, isocratic, } \\
\mathrm{RP}\end{array}$ & ChBuMWat (4:3:4:5) & & 0.55 & $\begin{array}{l}\text { Chen et al. } \\
2014\end{array}$ \\
\hline & $\begin{array}{lr}\text { Pedicularis } & \text { longiflora } \\
\text { Rudolph. var. } & \text { tubiformis } \\
\text { (Klotz) } & \text { Tsong } \\
\text { (Scrophulariaceae) } & \text { whole } \\
\text { plant } & \end{array}$ & $70 \% \mathrm{Et}$ & $120 \mathrm{mg}$ & $48.7 \mathrm{mg}$ & $98.1 \%$ & LLE & $\begin{array}{l}\mathrm{CCC} \text {, isocratic, } \\
\mathrm{RP}\end{array}$ & EBuWat (10:6:15) & & 4.11 & $\begin{array}{l}\text { Zhang et al. } \\
2015\end{array}$ \\
\hline & Plantago psyllium & $80 \% \mathrm{M}$ & $978 \mathrm{mg}$ & $165 \mathrm{mg}$ & $98.0 \%$ & LLE & isocratic, & EWat (1:1) & & 0.82 & $\mathrm{Li}$ et \\
\hline
\end{tabular}


Table 1 continued

\begin{tabular}{|c|c|c|c|c|c|c|c|c|c|c|c|}
\hline Compound & Species & $\begin{array}{l}\text { Extrac- } \\
\text { tion }\end{array}$ & $\begin{array}{l}\text { Sample } \\
\text { amount }\end{array}$ & $\begin{array}{l}\text { Isolated } \\
\text { amount }\end{array}$ & Purity & $\begin{array}{l}\text { Upstrea } \\
\text { m }\end{array}$ & CCS technique & CCS solvent system & $\begin{array}{l}\text { Downstre } \\
\text { am }\end{array}$ & $\begin{array}{l}\mathbf{K} \\
\text { value }\end{array}$ & References \\
\hline & (Plantaginaceae) seeds & & & & & & RP & & & & 2005 \\
\hline & $\begin{array}{l}\text { Cistanche deserticola Y.C. } \\
\text { Ma (Orobanchaceae) stems }\end{array}$ & $80 \% \mathrm{Et}$ & $\begin{array}{l}1412 \\
\mathrm{mg}\end{array}$ & $14.6 \mathrm{mg}$ & $92.5 \%$ & LLE & $\begin{array}{l}\mathrm{CCC}, \text { isocratic, } \\
\text { two-steps, RP }\end{array}$ & EEtWat $(5: 0.5: 4: 5)$ & & 0.87 & $\begin{array}{l}\mathrm{Li} \text { et al. } \\
2008\end{array}$ \\
\hline & $\begin{array}{lll}\text { Cistanche salsa } & \text { (C.A. } \\
\text { Mey.) } & \text { G. } & \text { Beck } \\
\text { (Orobanchaceae) stems }\end{array}$ & $75 \% \mathrm{Et}$ & $228 \mathrm{mg}$ & ns & $98.0 \%$ & $\begin{array}{l}\mathrm{BuOH} \\
\mathrm{L}-\mathrm{L} \\
\text { fraction }\end{array}$ & $\begin{array}{l}\text { CCC, isocratic, } \\
\text { two-steps, RP }\end{array}$ & EBuEtWat (4:0.6:0.6:5) & & - & $\begin{array}{l}\text { Lei et al. } \\
\text { 2001a }\end{array}$ \\
\hline & $\begin{array}{lr}\text { Cistanche } & \text { tubulosa } \\
\text { (Schrenk) } & \text { Wight }\end{array}$ & $\mathrm{M}^{*}$ & $214 \mathrm{mg}$ & ns & $95 \%$ & $\begin{array}{l}\text { Sephade } \\
\text { x LH-20 }\end{array}$ & $\begin{array}{l}\mathrm{CCC}, \text { isocratic, } \\
\text { two-steps, RP }\end{array}$ & $\begin{array}{l}\text { EBuEtWat }(35: 6: 6: 50 \\
\text { and 30:10:6:50) }\end{array}$ & & - & $\begin{array}{l}\text { Lei et al. } \\
2001 b\end{array}$ \\
\hline & $\begin{array}{l}\text { (Orobanchaceae) stems } \\
\text { Cistanche deserticola Y.C. } \\
\text { Ma (Orobanchaceae) stems }\end{array}$ & $60 \% \mathrm{Et}$ & $297 \mathrm{mg}$ & $30.9 \mathrm{mg}$ & $99.0 \%$ & $\begin{array}{l}\text { LLE + } \\
\text { SG }\end{array}$ & $\begin{array}{l}\mathrm{CCC} \text { isocratic, } \\
\mathrm{RP}\end{array}$ & EBuEtWat (4:0.6:0.6:5) & & 1.20 & $\begin{array}{l}\text { Han et al. } \\
2012\end{array}$ \\
\hline & $\begin{array}{lr}\text { Lamiophlomis } & \text { rotata } \\
\text { (Benth.) } & \text { Kudo }\end{array}$ & $65 \% \mathrm{Et}$ & $150 \mathrm{mg}$ & $41 \mathrm{mg}$ & $99.2 \%$ & $\begin{array}{l}\mathrm{LLE}+ \\
\mathrm{MR}\end{array}$ & $\begin{array}{l}\mathrm{CCC} \text {, isocratic, } \\
\mathrm{RP}\end{array}$ & EBuWat $(13: 3: 10)$ & & 1.96 & $\begin{array}{l}\text { Yue et al. } \\
2013 \mathrm{a}\end{array}$ \\
\hline & $\begin{array}{ll}\text { (Lamiaceaee) whole plant } \\
\text { Penstemon barbatus } \\
\text { (Cav.) Roth }\end{array}$ & $50 \% \mathrm{M}$ & ns & $79.6 \mathrm{mg}$ & $96.4 \%$ & MR AB8 & $\begin{array}{l}\mathrm{CCC}, \text { isocratic, } \\
\mathrm{RP}\end{array}$ & BuWat (1:1) & & 3.37 & $\begin{array}{l}\text { Xie et al. } \\
2012 \mathrm{a}\end{array}$ \\
\hline & $\begin{array}{lr}\text { (Plantaginaceae) leaves } \\
\text { Cistanche } & \text { tubulosa } \\
\text { (Schrenk) } & \text { Wight }\end{array}$ & $80 \% \mathrm{M}$ & $220 \mathrm{mg}$ & $5.1 \mathrm{mg}$ & $95.0 \%$ & - & $\begin{array}{l}\mathrm{CCC} \text {, isocratic, } \\
\mathrm{NP}\end{array}$ & $\begin{array}{l}\text { EBuWatAa } \\
(1: 1.2: 2: 0.2)\end{array}$ & & 1.11 & $\begin{array}{l}\text { Xie et al. } \\
2012 b\end{array}$ \\
\hline & $\begin{array}{l}\text { (Orobanchaceae) stens } \\
\text { Penstemon digitalis Nutt. } \\
\text { ex Sims (Plantaginaceae) } \\
\text { leaves }\end{array}$ & $50 \% \mathrm{M}$ & ns & $67.2 \mathrm{mg}$ & $92.6 \%$ & MR AB8 & $\begin{array}{l}\text { CCC, isocratic, } \\
\text { RP }\end{array}$ & BuWat (1:1) & & 3.37 & $\begin{array}{l}\text { Wang et al. } \\
2013\end{array}$ \\
\hline & $\begin{array}{lr}\text { Orobanche } & \text { rapum- } \\
\text { genistae } & \text { Thuill. } \\
\text { (Orobanchaceae) } & \text { whole } \\
\text { plant } & \end{array}$ & $70 \% \mathrm{Et}$ & $300 \mathrm{mg}$ & $30 \mathrm{mg}$ & $75 \%$ & & $\begin{array}{l}\mathrm{CCC}, \text { isocratic, } \\
\mathrm{RP} / \mathrm{NP}\end{array}$ & $\begin{array}{l}\text { EAcWat } \\
\text { RP: } 2.6: 2.6: 4.8 \\
\text { NP: }+2 \% \mathrm{M}\end{array}$ & & 0.55 & $\begin{array}{l}\text { Viron et al. } \\
1998\end{array}$ \\
\hline & $\begin{array}{l}\text { Rehmannia glutinosa } \\
\text { Libosch. (Orobanchaceae) } \\
\text { roots }\end{array}$ & $70 \% \mathrm{Et}$ & $165 \mathrm{mg}$ & $45 \mathrm{mg}$ & $96 \%$ & $\begin{array}{l}\text { MR } \\
\text { D101 }\end{array}$ & $\begin{array}{l}\mathrm{CCC} \text {, isocratic, } \\
\mathrm{RP}\end{array}$ & EBuWat (1:4:5) & & 3.10 & $\begin{array}{l}\text { Bu et al. } \\
2017\end{array}$ \\
\hline & $\begin{array}{lr}\text { Orobanche } & \text { rapum- } \\
\text { genistae } & \text { Thuill. } \\
\text { (Orobanchaceae) } & \text { whole } \\
\text { plant } & \end{array}$ & $70 \% \mathrm{Et}$ & $6 \mathrm{~g}$ & $1200 \mathrm{mg}$ & $80 \%$ & & $\begin{array}{l}\mathrm{CPC} \text {, isocratic, } \\
\mathrm{RP}\end{array}$ & EAtWat (10.6:1:9.7) & & - & $\begin{array}{l}\text { Viron et al. } \\
2000\end{array}$ \\
\hline & $\begin{array}{l}\text { Capraria biflora } \mathrm{L} \text {. } \\
\text { (Scrophulariaceae) aerial } \\
\text { parts }\end{array}$ & M & $15 \mathrm{~g}$ & $598 \mathrm{mg}$ & ns & & $\begin{array}{l}\mathrm{CPC}, \text { isocratic, } \\
\mathrm{NP}\end{array}$ & TetEWat (4:9:12) & & - & $\begin{array}{l}\text { Lemus et } \\
\text { al. } 2015\end{array}$ \\
\hline & $\begin{array}{l}\text { Plantago asiatica } \mathrm{L} \text {. } \\
\text { (Plantaginaceae) aerial } \\
\text { parts }\end{array}$ & $80 \% \mathrm{Et}$ & $\begin{array}{l}1341 \\
\mathrm{mg}\end{array}$ & $\begin{array}{l}293.8 \\
\mathrm{mg}\end{array}$ & $93.3 \%$ & LLE & $\begin{array}{l}\mathrm{CPC}, \text { isocratic, } \\
\mathrm{RP}\end{array}$ & $\begin{array}{l}\text { EBuEtWat } \\
(0.5: 0.5: 0.1: 1)\end{array}$ & & 2.69 & $\begin{array}{l}\mathrm{Li} \text { et al. } \\
2009\end{array}$ \\
\hline $\begin{array}{l}\text { Wiedemannioside } \\
\text { C }\end{array}$ & $\begin{array}{lr}\text { Pedicularis longiflora } \\
\text { Rudolph. var. } & \text { tubiformis } \\
\text { (Klotz) } & \text { Tong } \\
\text { (Scrophulariaceae) } & \text { whole } \\
\text { plant } & \\
\end{array}$ & $70 \% \mathrm{Et}$ & $120 \mathrm{mg}$ & $12.6 \mathrm{mg}$ & $97.9 \%$ & $\begin{array}{l}\mathrm{LLE} \\
\mathrm{MR}\end{array}$ & $\begin{array}{l}\text { CCC, isocratic, } \\
\text { RP }\end{array}$ & EBuWat (10:6:15) & & 0.39 & $\begin{array}{l}\text { Zhang et al. } \\
2015\end{array}$ \\
\hline
\end{tabular}

[C $\left.C_{6} M I M\right] B r$ 1-hexyl-3-methylimidazolium bromide, $A a$ acetic acid, Ac acetonitrile, At acetone, $B u$-butanol, $C C S$ countercurrent separation, $C E C C C$ recycling countercurrent chromatography, $C h$ chloroform, $C P C$ centrifugal partition chromatography, $D i$ dichloromethane, $E$ ethyl acetate, $E t$ ethanol, $C C C$ high-speed countercurrent chromatography, $H$-hexane, Iso isopropanol, $L L E$ liquid-liquid extraction, $M$ methanol, $M R$ macroporous resin, $N P$, normal-phase, $n s$ not specified, $P A$ polyamide, $R P$, reversed-phase, $S G$ silica gel, spHPLC semi-preparative high-performance liquid chromatography, Tet tetrahydrofuran, Wat water

${ }^{a}$ Methanol extract from faeces of Beagle dogs at $24 \mathrm{~h}$ after feeding with $3 \mathrm{~g} / \mathrm{kg} \mathrm{PhGs}$

purification of root extracts, solvent systems had 50\% water, $40 \%$ (on average) $n$-butanol and the rest was ethyl acetate, with only one or two PhGs isolated in a single run. Separation of up to five $\mathrm{PhGs}$ requires more non-polar solvent systems based on $45 \%$ water, $25-0 \%$ $n$-butanol, 25-50\% ethyl acetate and 5\% ethanol. The polarity of these systems is controlled by changing the ethyl acetate/n-butanol ratio. The large number of PhGs were isolated from extracts of "above ground" plant material with typical solvent ratios of $45-50 \%$ water, 30-50\% ethyl acetate and the rest is either $n$ butanol or ethanol/methanol. There was no clear correlation between solvent system polarity and $\mathrm{PhGs}$ classes based on the number of sugar moieties, partly because plants generally contain a range of $\mathrm{PhGs}$ of different types. However, the number of sugar units affect the elution order when present in the same extract, with triglycosides eluting first with more polar phase followed by diglycosides and then monoglycosides. 
Table 2 List of iridoids isolated through countercurrent separation

\begin{tabular}{|c|c|c|c|c|c|c|c|c|c|c|c|}
\hline Compound & Species & $\begin{array}{l}\text { Extrac- } \\
\text { tion }\end{array}$ & $\begin{array}{l}\text { Sample } \\
\text { amount }\end{array}$ & $\begin{array}{l}\text { Isolated } \\
\text { amount }\end{array}$ & Purity & $\begin{array}{l}\text { Upstrea } \\
\text { m }\end{array}$ & CCS technique & CCS solvent system & $\begin{array}{l}\text { Downstr } \\
\text { eam }\end{array}$ & $\begin{array}{l}\mathbf{K} \\
\text { value }\end{array}$ & References \\
\hline $\begin{array}{l}10 \text {-Hydroxy } \\
\text { hastatoside } \\
\text { 10-Hydroxycornin }\end{array}$ & $\begin{array}{l}\text { Cornus officinalis Sieb. Et } \\
\text { Zucc. (Cornaceae) fruits }\end{array}$ & $80 \%$ Et & $1 \mathrm{~g}$ & $\begin{array}{l}0.77 \mathrm{mg} \\
0.61 \mathrm{mg}\end{array}$ & $\begin{array}{l}88.4 \% \\
83.6 \%\end{array}$ & $\begin{array}{l}\text { MR } \\
\text { D101 }\end{array}$ & $\begin{array}{l}\text { CCC, isocratic } \\
\text { and EECCC, RP }\end{array}$ & BuWatAa $(5: 5: 1)$ & & $\begin{array}{l}4.52 \\
11.49\end{array}$ & $\begin{array}{ll}\text { Liang } & \text { et } \\
\text { al.2018 } & \end{array}$ \\
\hline $\begin{array}{l}\text { 3-Hydroxy } \\
\text { myopochlorin } \\
5 \text {-hydroxy } \\
\text { glutinoside }\end{array}$ & 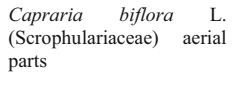 & M & $15 \mathrm{~g}$ & ns & ns & & $\begin{array}{l}\text { CPC, isocratic, } \\
\text { two-steps, NP }\end{array}$ & $\begin{array}{l}\text { TetEWat }(4: 9: 12) \text { and } \\
\text { EProWat }(8: 4: 12)\end{array}$ & SG & - & $\begin{array}{l}\text { Lemus et } \\
\text { al. } 2015\end{array}$ \\
\hline $6^{\prime}-O-\beta-\mathrm{D}-$ & Gentiana crassicaulis & $57.5 \%$ & $500 \mathrm{mg}$ & $12.8 \mathrm{mg}$ & $98.1 \%$ & & $\mathrm{CCC}$, isocratic, & $\operatorname{EtBuMAa}(1 \%)$ & & 1.75 & Liang et al. \\
\hline $\begin{array}{l}\text { Glucopyranosyl } \\
\text { gentiopicroside }\end{array}$ & $\begin{array}{l}\text { Duthie ex Burk. } \\
\text { (Gentianaceae) roots }\end{array}$ & Et & & & & & $\begin{array}{l}\text { step-flow } \\
\text { gradient, NP }\end{array}$ & $(0.5: 7.5: 0.5: 3.5)$ & & & $2013 b$ \\
\hline $\begin{array}{l}6-\beta \text {-Hydroxy } \\
\text { geniposide }\end{array}$ & $\begin{array}{l}\text { Gardenia jasminoides Ellis } \\
\text { (Rubiaceae) fruits }\end{array}$ & $40 \% \mathrm{Et}$ & $800 \mathrm{mg}$ & $52.2 \mathrm{mg}$ & $93.4 \%$ & $\begin{array}{l}\text { MR } \\
\text { HPD100 }\end{array}$ & $\begin{array}{l}\mathrm{CCC} \text {, isocratic, } \\
\mathrm{RP}\end{array}$ & BuEtWat $(10: 1: 10)$ & & 1.02 & $\begin{array}{l}\text { Wang et al. } \\
2015\end{array}$ \\
\hline $\begin{array}{l}6 \text { - } \beta \text {-Hydroxy } \\
\text { antirrhide }\end{array}$ & $\begin{array}{l}\text { Capraria biflora L. } \\
\text { (Scrophulariaceae) aerial } \\
\text { parts }\end{array}$ & M & $15 \mathrm{~g}$ & ns & ns & - & $\begin{array}{l}\text { CPC, isocratic, } \\
\text { two-steps, NP }\end{array}$ & $\begin{array}{l}\text { TetEtWat }(4: 9: 12) \text { and } \\
\text { EProWat }(8: 4: 12)\end{array}$ & SG & - & $\begin{array}{l}\text { Lemus et } \\
\text { al. } 2015\end{array}$ \\
\hline $\begin{array}{l}\text { 7-(S)- and 7-(R)-O- } \\
\text { ethylmorroniside }\end{array}$ & $\begin{array}{l}\text { Cornus officinalis Sieb. Et } \\
\text { Zucc. (Cornaceae) fruits }\end{array}$ & $80 \% \mathrm{Et}$ & $1 \mathrm{~g}$ & $\begin{array}{l}2.51 \mathrm{mg} \\
5.09 \mathrm{mg}\end{array}$ & $\begin{array}{l}89.5 \% \\
95.7 \%\end{array}$ & $\begin{array}{l}\text { MR } \\
\text { D101 }\end{array}$ & $\begin{array}{l}\mathrm{CCC} \text {, isocratic, } \\
\mathrm{RP}\end{array}$ & EMWatAa( $(5: 0.5: 5: 0.5)$ & & $\begin{array}{l}0.89 \\
1.32\end{array}$ & $\begin{array}{l}\text { Liang et al. } \\
2018\end{array}$ \\
\hline 7-Dehydrologanin & $\begin{array}{l}\text { Cornus officinalis Sieb. Et } \\
\text { Zucc. (Cornaceae) fruits }\end{array}$ & $80 \% \mathrm{Et}$ & $1 \mathrm{~g}$ & $4.51 \mathrm{mg}$ & $93.7 \%$ & $\begin{array}{l}\text { MR } \\
\text { D101 }\end{array}$ & $\begin{array}{l}\mathrm{CCC} \text {, isocratic, } \\
\mathrm{RP}\end{array}$ & BuWatAa $(5: 5: 1)$ & & 6.17 & $\begin{array}{l}\text { Liang et al. } \\
2018\end{array}$ \\
\hline $\begin{array}{l}\text { 7-O-(4-maloyl) } \\
\text { loganin }\end{array}$ & $\begin{array}{l}\text { Cornus officinalis Sieb. Et } \\
\text { Zucc. (Cornaceae) fruits }\end{array}$ & $80 \% \mathrm{Et}$ & $1 \mathrm{~g}$ & $2.22 \mathrm{mg}$ & $90.6 \%$ & $\begin{array}{l}\text { MR } \\
\text { D101 }\end{array}$ & $\begin{array}{l}\mathrm{CCC} \text {, isocratic, } \\
\mathrm{RP}\end{array}$ & EMWatAa( $(5: 0.5: 5: 0.5)$ & & 0.22 & $\begin{array}{l}\text { Liang et al. } \\
2018\end{array}$ \\
\hline $\begin{array}{l}\text { 7-O-(E-p- } \\
\text { coumaroyl)- and 7- } \\
O \text {-(E-caffeoyl)- } \\
\text { sylvestroside I }\end{array}$ & $\begin{array}{l}\text { Scabiosa stellata Cav. } \\
\text { (Caprifoliaceae) whole } \\
\text { plant }\end{array}$ & $70 \% \mathrm{Et}$ & $3 \mathrm{~g}$ & $\begin{array}{l}23 \mathrm{mg} \\
7 \mathrm{mg}\end{array}$ & ns & $\begin{array}{l}\text { MR } \\
\text { HP20 }\end{array}$ & $\begin{array}{l}\mathrm{CPC} \text {, isocratic, } \\
\mathrm{RP}\end{array}$ & TerAcWat(3:3:4) & RP-SG & - & $\begin{array}{l}\text { Lehbili et } \\
\text { al. } 2018\end{array}$ \\
\hline $\begin{array}{l}\text { 8-Hydroxy-10- } \\
\text { hydrosweroside }\end{array}$ & $\begin{array}{l}\text { Gentiana scabra Bunge } \\
\text { (Gentianaceae) roots }\end{array}$ & $95 \% \mathrm{Et}$ & $50 \mathrm{mg}$ & $8 \mathrm{mg}$ & $96.7 \%$ & LLE & $\begin{array}{l}\mathrm{CCC} \text {, isocratic, } \\
\mathrm{RP}\end{array}$ & $\begin{array}{l}\text { HBuMAa }(0.4 \%)(1.4: 8: \\
3: 15.5)\end{array}$ & & 0.29 & $\begin{array}{l}\text { Liang et al. } \\
2007\end{array}$ \\
\hline \multirow[t]{2}{*}{$\begin{array}{l}\text { 8-O- } \\
\text { acetylharpagide }\end{array}$} & $\begin{array}{l}\text { Capraria biflora L. } \\
\text { (Scrophulariaceae) aerial } \\
\text { parts }\end{array}$ & M & $15 \mathrm{~g}$ & ns & ns & & $\begin{array}{l}\text { CPC, isocratic, } \\
\text { two-steps, NP }\end{array}$ & $\begin{array}{l}\text { TetEWat( }(4: 9: 12) \\
\text { EProWat }(8: 4: 12)\end{array}$ & & - & $\begin{array}{l}\text { Lemus et } \\
\text { al. } 2015\end{array}$ \\
\hline & $\begin{array}{l}\text { Oxera coronata de Kok } \\
\text { (Lamiaceae) aerial parts }\end{array}$ & Wat & $347.3 \mathrm{~g}$ & $63.9 \mathrm{~g}$ & $\begin{array}{l}80- \\
90 \%\end{array}$ & & $\begin{array}{l}\mathrm{CPC} \text {, isocratic, } \\
\mathrm{NP}\end{array}$ & EProWat(3:2:5) & & - & $\begin{array}{l}\text { Remeur et } \\
\text { al. } 2017\end{array}$ \\
\hline Aucubin & $\begin{array}{l}\text { Aucuba japonica Thunb. } \\
\text { (Garryaceae) leaves }\end{array}$ & Wat & $105 \mathrm{~g}$ & $23.9 \mathrm{~g}$ & ns & & $\begin{array}{l}\text { CPC, isocratic, } \\
\text { NP }\end{array}$ & EProWat(10:3:7) & & 17.6 & $\begin{array}{l}\text { Markovic } \\
\text { et al. } 2014\end{array}$ \\
\hline Caprarioside & $\begin{array}{l}\text { Capraria biflora L. } \\
\text { (Scrophulariaceae) aerial } \\
\text { parts }\end{array}$ & M & $15 \mathrm{~g}$ & $16 \mathrm{mg}$ & ns & & $\begin{array}{l}\text { CPC, isocratic, } \\
\mathrm{NP}\end{array}$ & TetEWat(4:9:12) & & - & $\begin{array}{l}\text { Lemus et } \\
\text { al. } 2015\end{array}$ \\
\hline \multirow[t]{2}{*}{ Catalpol } & $\begin{array}{l}\text { Rehmannia glutinosa } \\
\text { Libosch. } \\
\text { (Scrophulariaceae) roots }\end{array}$ & $75 \% \mathrm{Et}$ & $105 \mathrm{mg}$ & $35 \mathrm{mg}$ & $95.6 \%$ & $\begin{array}{l}\text { LLE } \\
\text { SG }\end{array}$ & $\begin{array}{l}\mathrm{CCC} \text {, isocratic, } \\
\mathrm{NP}\end{array}$ & EBuWat $(2: 1: 3)$ & & 0.51 & $\begin{array}{l}\text { Tong et al. } \\
2015\end{array}$ \\
\hline & $\begin{array}{l}\text { Capraria biflora } \text { L. } \\
\text { (Scrophulariaceae) aerial } \\
\text { parts }\end{array}$ & M & $15 \mathrm{~g}$ & $\mathrm{~ns}$ & $\mathrm{~ns}$ & & $\begin{array}{l}\text { CPC, isocratic, } \\
\text { two-steps, NP }\end{array}$ & $\begin{array}{l}\text { TetEWat( }(4: 9: 12) \\
\text { EProWat }(8: 4: 12)\end{array}$ & SG & - & $\begin{array}{l}\text { Lemus et } \\
\text { al. } 2015\end{array}$ \\
\hline Catalposide & $\begin{array}{ll}\text { Veronica ciliata } & \text { Fisch. } \\
\text { (Scrophulariaceae) } & \text { aerial } \\
\text { parts } & \end{array}$ & $95 \% \mathrm{Et}$ & $200 \mathrm{mg}$ & $10 \mathrm{mg}$ & $99.0 \%$ & LLE & $\begin{array}{l}\text { CCC, isocratic, } \\
\text { NP }\end{array}$ & HBuWat (1.5:5:5) & & 0.52 & $\begin{array}{l}\mathrm{Lu} \text { et al. } \\
2016\end{array}$ \\
\hline Chlorotuberside & $\begin{array}{lr}\text { Lamiophlomis } & \text { rotata } \\
\text { (Benth.) } & \text { Kudo } \\
\text { (Lamiaceae) ns } & \end{array}$ & $65 \% \mathrm{Et}$ & $150 \mathrm{mg}$ & $27 \mathrm{mg}$ & $97.3 \%$ & $\begin{array}{l}\text { LLE + } \\
\text { MR } \\
\text { D101 }\end{array}$ & $\begin{array}{l}\mathrm{CCC} \text {, isocratic, } \\
\mathrm{RP}\end{array}$ & EBuWat $(5: 14: 12)$ & & 0.63 & $\begin{array}{l}\text { Yue et al. } \\
2013 \text { b }\end{array}$ \\
\hline Cornusides I and II & $\begin{array}{l}\text { Cornus officinalis Sieb. Et } \\
\text { Zucc. (Cornaceae) fruits }\end{array}$ & $80 \% \mathrm{Et}$ & $1 \mathrm{~g}$ & $\begin{array}{l}4.78 \mathrm{mg} \\
29.4 \mathrm{mg}\end{array}$ & $\begin{array}{l}92.3 \% \\
97.4 \%\end{array}$ & $\begin{array}{l}\text { MR } \\
\text { D101 }\end{array}$ & $\begin{array}{l}\text { CCC, EECCC, } \\
\text { RP }\end{array}$ & EMWatAa $(5: 0.5: 5: 0.5)$ & & $\begin{array}{l}0.54 \\
1.99\end{array}$ & $\begin{array}{l}\text { Liang et al. } \\
2018\end{array}$ \\
\hline $\begin{array}{l}\text { Deglucoserrulatosi } \\
\text { de }\end{array}$ & $\begin{array}{l}\text { Gentiana macrophylla Pall } \\
\text { (Gentianaceae) ns }\end{array}$ & Wat & ns & $37 \mathrm{mg}$ & $95.3 \%$ & & $\begin{array}{l}\mathrm{CCC} \text {, isocratic, } \\
\mathrm{RP}\end{array}$ & EBuWatAa $(2: 3: 5: 0.6)$ & spHPLC & - & $\begin{array}{l}\text { Wu et al. } \\
2012\end{array}$ \\
\hline $\begin{array}{l}\text { Eustomorusside } \\
\text { Eustomoside }\end{array}$ & $\begin{array}{l}\text { Scabiosa stellata Cav. } \\
\text { (Caprifoliaceae) } \\
\text { plant }\end{array}$ & $70 \% \mathrm{Et}$ & $3 g$ & $\begin{array}{l}6 \mathrm{mg} \\
5 \mathrm{mg}\end{array}$ & ns & $\begin{array}{l}\text { MR } \\
\text { HP20 }\end{array}$ & $\begin{array}{l}\mathrm{CPC} \text {, isocratic, } \\
\mathrm{RP}\end{array}$ & TerAcWat(3:3:4) & SG & - & $\begin{array}{l}\text { Lehbili et } \\
\text { al. } 2018\end{array}$ \\
\hline Gardenoside & $\begin{array}{l}\text { Gardenia jasminoides Ellis } \\
\text { (Rubiaceae) fruits }\end{array}$ & $40 \% \mathrm{Et}$ & $800 \mathrm{mg}$ & $151.1 \mathrm{mg}$ & $91.7 \%$ & $\begin{array}{l}\text { MR } \\
\text { HPD100 }\end{array}$ & $\begin{array}{l}\mathrm{CCC} \text {, isocratic, } \\
\mathrm{RP}\end{array}$ & BuEtWat $(10: 1: 10)$ & & 0.75 & $\begin{array}{l}\text { Wang et al. } \\
2015\end{array}$ \\
\hline \multirow[t]{5}{*}{ Geniposide } & $\begin{array}{l}\text { Gardenia jasminoides Ellis } \\
\text { (Rubiaceae) fruits }\end{array}$ & $40 \% \mathrm{Et}$ & $800 \mathrm{mg}$ & $587.2 \mathrm{mg}$ & $98.2 \%$ & $\begin{array}{l}\text { MR } \\
\text { HPD100 }\end{array}$ & $\begin{array}{l}\text { CCC, isocratic, } \\
\text { RP }\end{array}$ & EBuWat $(2: 1.5: 3)$ & & 0.70 & $\begin{array}{l}\text { Wang et al. } \\
2015\end{array}$ \\
\hline & $\begin{array}{l}\text { Gardenia jasminoides Ellis } \\
\text { (Rubiaceae) fruits }\end{array}$ & $50 \% \mathrm{Et}$ & $1 \mathrm{~g}$ & $389 \mathrm{mg}$ & $98.0 \%$ & $\begin{array}{l}\text { MR } \\
\text { D101 }\end{array}$ & $\begin{array}{l}\mathrm{CCC} \text {, isocratic, } \\
\mathrm{RP}\end{array}$ & EBuWat $(2: 1.5: 3)$ & & 0.40 & $\begin{array}{l}\text { Zhou et al. } \\
2005\end{array}$ \\
\hline & $\begin{array}{l}\text { Gardenia jasminoides Ellis } \\
\text { (Rubiaceae) fruits }\end{array}$ & $80 \% \mathrm{Et}$ & $150 \mathrm{mg}$ & $43 \mathrm{mg}$ & $98.7 \%$ & LLE & $\begin{array}{l}\mathrm{CCC} \text {, isocratic, } \\
\mathrm{RP}\end{array}$ & EBuWat (1:4:5) & & 0.31 & $\begin{array}{l}\text { Liang et al. } \\
2014\end{array}$ \\
\hline & $\begin{array}{l}\text { Gardenia jasminoides Ellis } \\
\text { (Rubiaceae) fruits }\end{array}$ & Wat & $4 \mathrm{~g}$ & $445 \mathrm{mg}$ & $95.2 \%$ & $\begin{array}{l}\text { MR } \\
\text { HPD100 }\end{array}$ & $\begin{array}{l}\mathrm{CCC} \text {, isocratic, } \\
\mathrm{RP}\end{array}$ & EBuWat (1:4:5) & & 0.93 & $\begin{array}{l}\text { Zhang et al. } \\
2012\end{array}$ \\
\hline & $\begin{array}{l}\text { Gardenia jasminoides Ellis } \\
\text { (Rubiaceae) fruits }\end{array}$ & $80 \% \mathrm{M}$ & $500 \mathrm{mg}$ & $56.2 \mathrm{mg}$ & $95 \%$ & & $\begin{array}{l}\text { CPC, isocratic, } \\
\text { RP }\end{array}$ & EIsoWat $(3: 2: 5)$ & & - & $\begin{array}{l}\text { Kim and } \\
\text { Kim } 2007\end{array}$ \\
\hline \multirow[t]{3}{*}{ Geniposidic acid } & $\begin{array}{l}\text { Gardenia jasminoides Ellis } \\
\text { (Rubiaceae) fruits }\end{array}$ & $40 \% \mathrm{Et}$ & $800 \mathrm{mg}$ & $24.5 \mathrm{mg}$ & $92.5 \%$ & $\begin{array}{l}\text { MR } \\
\text { HPD100 }\end{array}$ & $\begin{array}{l}\mathrm{CCC} \text {, isocratic, } \\
\mathrm{RP}\end{array}$ & BuEtWat $(10: 1: 10)$ & & 1.84 & $\begin{array}{l}\text { Wang et al. } \\
2015\end{array}$ \\
\hline & $\begin{array}{l}\text { Gardenia jasminoides Ellis } \\
\text { (Rubiaceae) fruits }\end{array}$ & $80 \% \mathrm{Et}$ & $150 \mathrm{mg}$ & $4 \mathrm{mg}$ & $90.4 \%$ & LLE & $\begin{array}{l}\mathrm{CCC} \text {, isocratic, } \\
\mathrm{RP}\end{array}$ & EBuWat (1:4:5) & & 2.41 & $\begin{array}{l}\text { Liang et al. } \\
2014\end{array}$ \\
\hline & $\begin{array}{l}\text { Eucommia ulmoides Oliv. } \\
\text { (Eucommiaceae) leaves }\end{array}$ & Wat & $80 \mathrm{mg}$ & $41.2 \mathrm{mg}$ & $99.2 \%$ & $\begin{array}{l}\mathrm{LLE} \mathrm{+} \\
\mathrm{MR} \\
\mathrm{D} 101+ \\
\text { carbon }\end{array}$ & $\begin{array}{l}\mathrm{CCC} \text {, isocratic, } \\
\mathrm{RP}\end{array}$ & EBuWat $(1: 2: 3)$ & & 0.76 & $\begin{array}{l}\text { Dai et al. } \\
2013\end{array}$ \\
\hline Gentiopicroside & $\begin{array}{l}\text { Gentiana scabra Bunge } \\
\text { (Gentianaceae) roots }\end{array}$ & Et & $300 \mathrm{mg}$ & $136 \mathrm{mg}$ & $99.6 \%$ & MR & $\begin{array}{l}\mathrm{CCC} \text {, isocratic, } \\
\mathrm{RP}\end{array}$ & EBuWat $(2: 1: 3)$ & & - & $\begin{array}{l}\mathrm{Xu} \text { et al. } \\
2007\end{array}$ \\
\hline
\end{tabular}


Table 2 continued

\begin{tabular}{|c|c|c|c|c|c|c|c|c|c|c|c|}
\hline Compound & Species & $\begin{array}{l}\text { Extrac- } \\
\text { tion }\end{array}$ & $\begin{array}{l}\text { Sample } \\
\text { amount }\end{array}$ & $\begin{array}{l}\text { Isolated } \\
\text { amount }\end{array}$ & Purity & $\begin{array}{l}\text { Upstrea } \\
\text { m }\end{array}$ & CCS technique & CCS solvent system & $\begin{array}{l}\text { Downstr } \\
\text { eam }\end{array}$ & $\begin{array}{l}\mathrm{K} \\
\text { value }\end{array}$ & References \\
\hline & $\begin{array}{l}\text { Gentiana scabra Bunge } \\
\text { (Gentianaceae) roots }\end{array}$ & & ns & ns & $97.0 \%$ & & $\begin{array}{l}\text { CCC, isocratic, } \\
\text { NP }\end{array}$ & ChTerMWat $(5: 2: 4: 4)$ & & - & $\begin{array}{l}\text { Wang et al. } \\
2007\end{array}$ \\
\hline & $\begin{array}{l}\text { Gentiana scabra Bunge } \\
\text { (Gentianaceae) roots }\end{array}$ & M & $100 \mathrm{mg}$ & $35.66 \mathrm{mg}$ & $98.6 \%$ & LLE & $\begin{array}{l}\mathrm{CCC} \text {, isocratic, } \\
\mathrm{RP}\end{array}$ & EBuWat $(2: 1: 3)$ & & 0.70 & $\begin{array}{l}\text { Chen et al. } \\
2017\end{array}$ \\
\hline & $\begin{array}{l}\text { Swertia mussotii Franch. } \\
\text { (Gentianaceae) } \\
\text { plant }\end{array}$ & $75 \% \mathrm{Et}$ & $300 \mathrm{mg}$ & $75 \mathrm{mg}$ & $99.6 \%$ & LLE & $\begin{array}{l}\mathrm{CCC} \text {, isocratic, } \\
\mathrm{RP}\end{array}$ & ChBuMWat $(4: 2: 3: 3)$ & $\begin{array}{l}\text { Sephade } \\
\text { x LH-20 }\end{array}$ & 0.72 & $\begin{array}{l}\text { Chen et al. } \\
2015\end{array}$ \\
\hline & $\begin{array}{l}\text { Swertia franchetiana H. } \\
\text { Smith (Gentianaceae) ns }\end{array}$ & ns & $100 \mathrm{mg}$ & $14 \mathrm{mg}$ & ns & & $\mathrm{CCC}$, isocratic & ChMWat $(4: 4: 2)$ & & - & $\begin{array}{l}\text { Cheng et } \\
\text { al. } 2010\end{array}$ \\
\hline & $\begin{array}{l}\text { Gentiana macrophylla } \\
\text { Pall. (Gentianaceae) aerial } \\
\text { parts }\end{array}$ & $95 \% \mathrm{Et}$ & $300 \mathrm{mg}$ & $150 \mathrm{mg}$ & $94 \%$ & & $\mathrm{CCC}$, isocratic & ChMWat (4:4:2) & & - & $\begin{array}{l}\text { Huangfu et } \\
\text { al. } 2007\end{array}$ \\
\hline & Gentiana crassicaulis & $57.5 \%$ & $500 \mathrm{mg}$ & $40.7 \mathrm{mg}$ & $99.4 \%$ & & CCC, step-flow & $\operatorname{EBuMAa}(1 \%)$ & & 1.06 & Liang et al. \\
\hline & $\begin{array}{l}\text { Duthie ex Burk. } \\
\text { (Gentianaceae) roots }\end{array}$ & & & & & & gradient, NP & $(0.5: 7.5: 0.5: 3.5)$ & & & $2013 b$ \\
\hline & $\begin{array}{l}\text { Gentiana macrophylla } \\
\text { Pall. (Gentianaceae) aerial } \\
\text { parts }\end{array}$ & Wat & ns & $52.3 \mathrm{mg}$ & $99.2 \%$ & & $\mathrm{CCC}$, isocratic & EBuWatAa (2:3:5:0.6) & spHPLC & - & $\begin{array}{l}\text { Wu et al. } \\
2012\end{array}$ \\
\hline & $\begin{array}{l}\text { Gentiana macrophylla } \\
\text { Pall. (Gentianaceae) roots }\end{array}$ & M & $750 \mathrm{mg}$ & $348 \mathrm{mg}$ & $97 \%$ & LLE & $\begin{array}{l}\text { CCC, step-flow } \\
\text { gradient, NP }\end{array}$ & $\operatorname{BuTfa}(0.1 \%)(1: 1)$ & & 1.56 & $\begin{array}{l}\text { Rho et al. } \\
2014\end{array}$ \\
\hline & $\begin{array}{l}\text { Centaurium erythraea } \\
\text { Rafn. (Gentianaceae) aerial } \\
\text { parts }\end{array}$ & M & $4 \mathrm{~g}$ & $13 \mathrm{mg}$ & ns & & $\begin{array}{l}\text { CPC, isocratic, } \\
\text { NP }\end{array}$ & EEtWat $(7.5: 3: 5)$ & spHPLC & - & $\begin{array}{l}\text { Mandova et } \\
\text { al. } 2017\end{array}$ \\
\hline \multirow[t]{3}{*}{ Harpagoside } & $\begin{array}{l}\text { Scrophularia ningpoensis } \\
\text { Hemsl. (Scrophulariaceae) } \\
\text { roots }\end{array}$ & $90 \% \mathrm{Et}$ & $276 \mathrm{mg}$ & $11 \mathrm{mg}$ & $97.0 \%$ & LLE & $\begin{array}{l}\mathrm{CCC} \text {, isocratic, } \\
\mathrm{RP}\end{array}$ & EBuWat $(9: 1: 10)$ & & 1.01 & $\begin{array}{l}\text { Tong et al. } \\
2006\end{array}$ \\
\hline & $\begin{array}{l}\text { Scrophularia ningpoensis } \\
\text { Hemsl. (Scrophulariaceae) } \\
\text { roots }\end{array}$ & $70 \% \mathrm{Et}$ & $200 \mathrm{mg}$ & $22 \mathrm{mg}$ & $98.0 \%$ & $\begin{array}{l}\text { MR } \\
\text { D101 }\end{array}$ & $\begin{array}{l}\mathrm{CCC} \text {, isocratic, } \\
\mathrm{NP}\end{array}$ & ChBuMWat $(4: 1: 3: 2)$ & & 1.05 & $\begin{array}{l}\text { Tian et al. } \\
2012\end{array}$ \\
\hline & $\begin{array}{l}\text { Scrophularia ningpoensis } \\
\text { Hemsl. (Scrophulariaceae) } \\
\text { roots }\end{array}$ & $90 \% \mathrm{Et}$ & $311 \mathrm{mg}$ & $14 \mathrm{mg}$ & $96.5 \%$ & LLE & $\begin{array}{l}\mathrm{CCC}, \quad \text { dual } \\
\text { mode, } \mathrm{RP} / \mathrm{NP}\end{array}$ & EBuWatAa $(1: 8: 10: 1)$ & & 6.20 & $\begin{array}{l}\text { Tong et al. } \\
2009\end{array}$ \\
\hline Ipolamiide & $\begin{array}{l}\text { Stachytarpheta } \\
\text { cayennensis (Rich.) Vahl. } \\
\text { (Verbenaceae) roots }\end{array}$ & Et & $1 \mathrm{~g}$ & $73 \mathrm{mg}$ & ns & LLE & $\begin{array}{l}\text { CCC, step- } \\
\text { gradient, NP }\end{array}$ & $\begin{array}{l}\text { EBuWat }(1: x: 1, x=0.05- \\
\text { 1) }\end{array}$ & & - & $\begin{array}{l}\text { Leitao et al. } \\
2005\end{array}$ \\
\hline Isovogeloside & $\begin{array}{l}\text { Halenia campanulata } \\
\text { Cuatrec. (Gentianaceae) ns }\end{array}$ & M & $240 \mathrm{mg}$ & $24 \mathrm{mg}$ & ns & SG & $\begin{array}{l}\mathrm{CCC} \text {, isocratic, } \\
\mathrm{NP}\end{array}$ & ChMWat (9:12:8) & & - & $\begin{array}{l}\text { Hostettman } \\
\text { and } \\
\text { Marston } \\
2001\end{array}$ \\
\hline Kingiside & $\begin{array}{l}\text { Cornus officinalis Sieb. et } \\
\text { Zucc. (Cornaceae) fruits }\end{array}$ & $80 \% \mathrm{Et}$ & $1 \mathrm{~g}$ & $5.2 \mathrm{mg}$ & $91.6 \%$ & $\begin{array}{l}\text { MR } \\
\text { D101 }\end{array}$ & $\begin{array}{l}\mathrm{CCC}, \text { isocratic, } \\
\mathrm{RP}\end{array}$ & BuWatAa $(5: 5: 1)$ & & 0.38 & $\begin{array}{l}\text { Liang et al. } \\
2018\end{array}$ \\
\hline Ligstroside & $\begin{array}{l}\text { Olea europea L. } \\
\text { (Oleaceae) leaves }\end{array}$ & $60 \% \mathrm{Et}$ & $120 \mathrm{mg}$ & ns & $93.0 \%$ & MR AB8 & $\begin{array}{l}\mathrm{CCC} \text {, isocratic, } \\
\mathrm{RP}\end{array}$ & EWat $(1: 1)$ & spHPLC & 0.74 & $\begin{array}{l}\text { Zhang et al. } \\
2014\end{array}$ \\
\hline Loganetin & $\begin{array}{l}\text { Alstonia scholaris } \text { (L.) } \\
\text { R.Br. (Apocynaceae) stem } \\
\text { bark }\end{array}$ & M & $1.5 \mathrm{~g}$ & $48 \mathrm{mg}$ & $94.4 \%$ & LLE & $\begin{array}{l}\mathrm{CPC} \text {, isocratic, } \\
\mathrm{NP}\end{array}$ & $\begin{array}{l}\text { TerAcWat }(3: 1.5: 3)+ \\
\mathrm{HCl}+\text { TEA }\end{array}$ & & - & $\begin{array}{l}\text { Maurya et } \\
\text { al. } 2014\end{array}$ \\
\hline \multirow[t]{5}{*}{ Loganic acid } & $\begin{array}{l}\text { Gentiana scabra Bunge } \\
\text { (Gentianaceae) roots }\end{array}$ & M & $100 \mathrm{mg}$ & 4.36 & $98.1 \%$ & LLE & & EBuWat $(2: 1: 3)$ & & 0.41 & $\begin{array}{l}\text { Chen et al. } \\
2017\end{array}$ \\
\hline & $\begin{array}{l}\text { Gentiana crassicaulis } \\
\text { Duthie ex Burk. } \\
\text { (Gentianaceae) roots }\end{array}$ & $\begin{array}{l}57.5 \% \\
\text { Et }\end{array}$ & $500 \mathrm{mg}$ & $16.3 \mathrm{mg}$ & $98.6 \%$ & - & $\begin{array}{l}\mathrm{CCC} \text {, isocratic, } \\
\mathrm{RP}\end{array}$ & $\begin{array}{l}\text { EBuMAa( } 1 \%) \\
(0.5: 7.5: 0.5: 3.5)\end{array}$ & & 2.82 & $\begin{array}{l}\text { Liang et al. } \\
2013 b\end{array}$ \\
\hline & $\begin{array}{l}\text { Gentiana macrophylla Pall } \\
\text { (Gentianaceae) aerial parts }\end{array}$ & $95 \% \mathrm{Et}$ & $600 \mathrm{mg}$ & $22.4 \mathrm{mg}$ & $90.2 \%$ & & $\mathrm{CCC}$, isocratic & EBuWatAa $(2: 3: 5: 0.6)$ & spHPLC & 0.24 & $\begin{array}{l}\text { Wu et al. } \\
2012\end{array}$ \\
\hline & $\begin{array}{l}\text { Gentiana macrophylla } \\
\text { Pallas (Gentianaceae) roots }\end{array}$ & M & $750 \mathrm{mg}$ & $54 \mathrm{mg}$ & $97.0 \%$ & LLE & $\begin{array}{l}\mathrm{CCC} \text {, step-flow } \\
\text { gradient, NP }\end{array}$ & $\operatorname{BuTfa}(0.1 \%)(1: 1)$ & & 4.76 & $\begin{array}{l}\text { Rho et al. } \\
2014\end{array}$ \\
\hline & $\begin{array}{l}\text { Cornus officinalis Sieb. et } \\
\text { Zucc. (Cornaceae) fruits }\end{array}$ & $80 \% \mathrm{Et}$ & $1 \mathrm{~g}$ & $5.8 \mathrm{mg}$ & $91.1 \%$ & $\begin{array}{l}\text { MR } \\
\text { D101 }\end{array}$ & $\begin{array}{l}\text { CCC, isocratic, } \\
\text { RP }\end{array}$ & $\operatorname{BuWat} \mathrm{Aa}(5: 5: 1)$ & & 1.36 & $\begin{array}{l}\text { Liang et al. } \\
2018\end{array}$ \\
\hline \multirow[t]{3}{*}{ Loganin } & $\begin{array}{l}\text { Cornus officinalis Sieb. et } \\
\text { Zucc. (Cornaceae) fruits }\end{array}$ & $75 \% \mathrm{Et}$ & $100 \mathrm{mg}$ & $12.6 \mathrm{mg}$ & $98.6 \%$ & $\begin{array}{l}\text { D101 } \\
\text { MR }\end{array}$ & $\begin{array}{l}\mathrm{CCC} \text {, isocratic, } \\
\mathrm{NP}\end{array}$ & $\begin{array}{l}\text { DiBuMWatAa } \\
(5: 2: 5: 4: 0.1)\end{array}$ & & 0.76 & $\begin{array}{l}\text { Liang et al. } \\
2013 \mathrm{a}\end{array}$ \\
\hline & $\begin{array}{l}\text { Cornus officinalis Sieb. et } \\
\text { Zucc. (Cornaceae) fruits }\end{array}$ & $50 \% \mathrm{M}$ & $50 \mathrm{mg}$ & $11.5 \mathrm{mg}$ & 98.6 & $\begin{array}{l}\text { D101 } \\
\text { MR }\end{array}$ & $\begin{array}{l}\mathrm{CCC} \text {, isocratic, } \\
\mathrm{RP}\end{array}$ & BuMAa(1\%) (4:1:6) & & 1.30 & $\begin{array}{l}\text { Liu et al. } \\
2009\end{array}$ \\
\hline & $\begin{array}{l}\text { Cornus officinalis Sieb. et } \\
\text { Zucc. (Cornaceae) fruits }\end{array}$ & $80 \% \mathrm{Et}$ & $1 \mathrm{~g}$ & $55.8 \mathrm{mg}$ & $97.2 \%$ & $\begin{array}{l}\text { MR } \\
\text { D101 }\end{array}$ & $\begin{array}{l}\mathrm{CCC}, \text { isocratic, } \\
\mathrm{EECCC}, \mathrm{RP}\end{array}$ & BuWatAa $(5: 5: 1)$ & & 15.17 & $\begin{array}{l}\text { Liang et al. } \\
2018\end{array}$ \\
\hline Macfadyenoside & $\begin{array}{l}\text { Capraria biflora L. } \\
\text { (Scrophulariaceae) aerial } \\
\text { parts }\end{array}$ & M & $15 \mathrm{~g}$ & $109 \mathrm{mg}$ & ns & & $\begin{array}{l}\mathrm{CCC} \text {, isocratic, } \\
\text { two-steps, NP }\end{array}$ & $\begin{array}{l}\text { TetEWat }(4: 9: 12) \text { and } \\
\text { EProWat }(8: 4: 12)\end{array}$ & & - & $\begin{array}{l}\text { Lemus et } \\
\text { al. } 2015\end{array}$ \\
\hline \multirow[t]{3}{*}{ Morroniside } & $\begin{array}{l}\text { Cornus officinalis Sieb. et } \\
\text { Zucc. (Cornaceae) fruits }\end{array}$ & $75 \% \mathrm{Et}$ & $28.5 \mathrm{mg}$ & $28.5 \mathrm{mg}$ & $99.1 \%$ & $\begin{array}{l}\text { D101 } \\
\text { MR }\end{array}$ & $\begin{array}{l}\mathrm{CCC} \text {, isocratic, } \\
\mathrm{NP}\end{array}$ & $\begin{array}{l}\text { DiBuMWatAa } \\
(5: 2: 5: 4: 0.1)\end{array}$ & & 1.57 & $\begin{array}{l}\text { Liang et al. } \\
2013 a\end{array}$ \\
\hline & $\begin{array}{l}\text { Cornus officinalis Sieb. et } \\
\text { Zucc. (Cornaceae) fruits }\end{array}$ & $50 \% \mathrm{M}$ & $50 \mathrm{mg}$ & $28.7 \mathrm{mg}$ & $97.8 \%$ & $\begin{array}{l}\text { D101 } \\
\text { MR }\end{array}$ & $\begin{array}{l}\mathrm{CCC} \text {, isocratic, } \\
\mathrm{RP}\end{array}$ & $\operatorname{BuMAa}(1 \%)(4: 1: 6)$ & & 0.74 & $\begin{array}{l}\text { Liu et al. } \\
2009\end{array}$ \\
\hline & $\begin{array}{l}\text { Cornus officinalis Sieb. et } \\
\text { Zucc. (Cornaceae) fruits }\end{array}$ & $80 \% \mathrm{Et}$ & $1 \mathrm{~g}$ & $68.8 \mathrm{mg}$ & $98.7 \%$ & $\begin{array}{l}\text { MR } \\
\text { D101 }\end{array}$ & $\begin{array}{l}\mathrm{CCC} \text {, isocratic, } \\
\mathrm{RP}\end{array}$ & BuWatAa $(5: 5: 1)$ & & 0.84 & $\begin{array}{l}\text { Liang et al. } \\
2018\end{array}$ \\
\hline \multirow[t]{3}{*}{ Oleuropein } & $\begin{array}{l}\text { Olea europea L. } \\
\text { (Oleaceae) leaves }\end{array}$ & $60 \% \mathrm{Et}$ & $120 \mathrm{mg}$ & ns & $91 \%$ & MR AB8 & $\begin{array}{l}\mathrm{CCC} \text {, isocratic, } \\
\mathrm{RP}\end{array}$ & EWat $(1: 1)$ & spHPLC & 0.66 & $\begin{array}{l}\text { Zhang et al. } \\
2014\end{array}$ \\
\hline & $\begin{array}{l}\text { Cynomorium songaricum } \\
\text { Rupr. (Cynomoriaceae) } \\
\text { stems }\end{array}$ & Wat & $600 \mathrm{mg}$ & $11.3 \mathrm{mg}$ & $95.6 \%$ & MR & $\begin{array}{l}\mathrm{CCC} \text {, isocratic, } \\
\mathrm{RP}\end{array}$ & EWat $(1: 1)$ & & 1.17 & $\begin{array}{l}\text { Wang et al. } \\
2016\end{array}$ \\
\hline & $\begin{array}{l}\text { Olea europea L. } \\
\text { (Oleaceae) leaves }\end{array}$ & Wat & ns & $25.4 \mathrm{mg}$ & $95 \%$ & & $\begin{array}{l}\mathrm{CPC} \text {, isocratic, } \\
\mathrm{RP}\end{array}$ & HEEtWat (1:9:1:9) & & 0.71 & $\begin{array}{l}\text { Boka et al. } \\
2015\end{array}$ \\
\hline Penstemonoside & Lamiophlomis rotata & $65 \% \mathrm{Et}$ & $150 \mathrm{mg}$ & $21 \mathrm{mg}$ & $99.3 \%$ & LLE & $\mathrm{CCC}$, isocratic, & EBuWat (5:14:12) & & 1.03 & Yue et al. \\
\hline
\end{tabular}


Table 2 continued

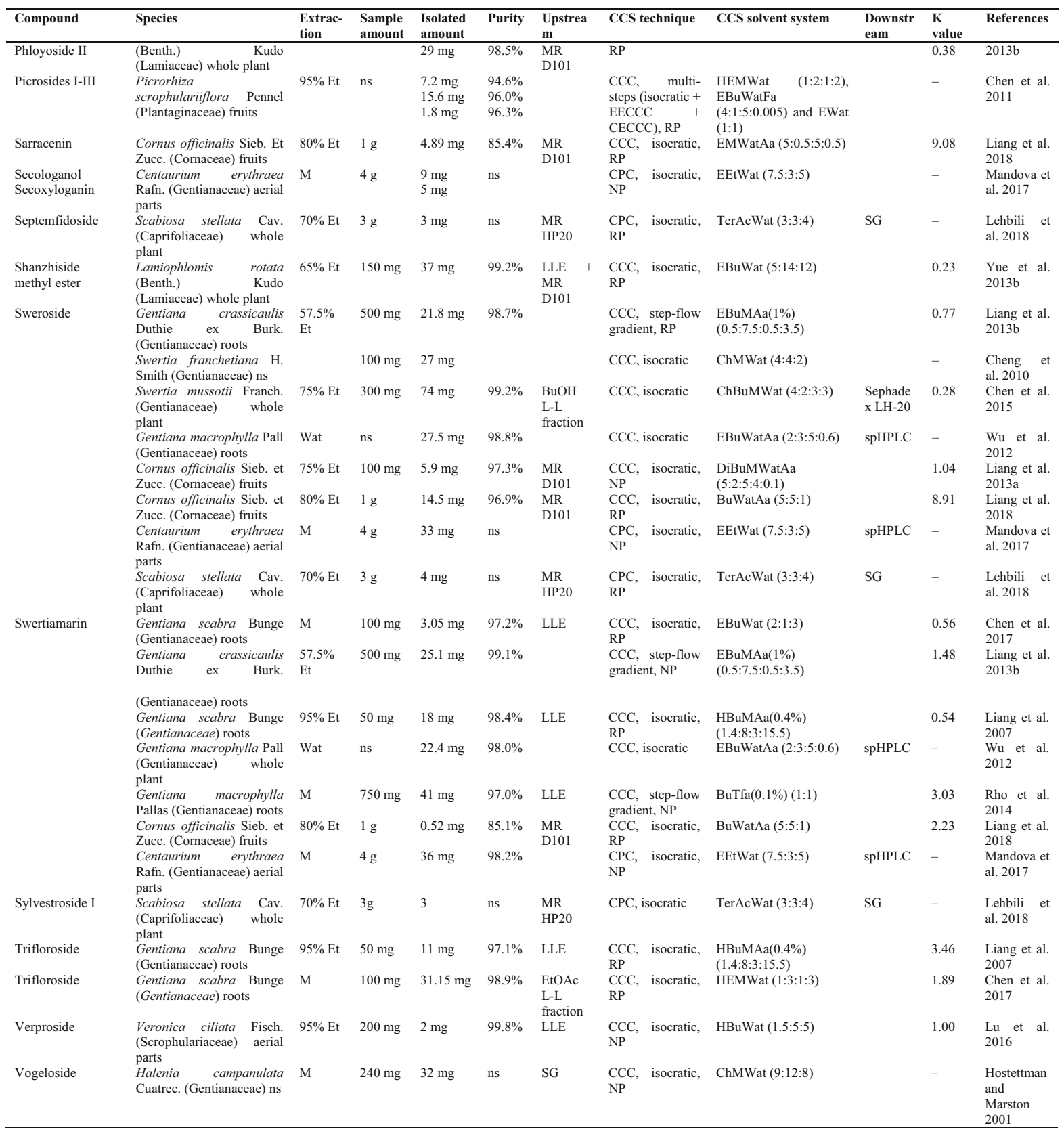

$A a$ acetic acid, $A c$ acetronitrile, $B u$ n-butanol, $C C S$ countercurrent separation, $C E C C C$ recycling countercurrent chromatography, $C h$ chloroform, $C P C$ centrifugal partition chromatography, $D i$ dichloromethane, EECCC elution-extrusion countercurrent chromatography, $E$ ethyl acetate, Et ethanol, $F a$ formic acid, $C C C$ high-speed countercurrent chromatography, $H n$-hexane, Iso isopropanol, $L L E$ liquid-liquid extraction, $M$ methanol, $M R$ macroporous resin, Ter methyl $t$-buthyl ether, NP normal-phase Pro $n$ propanol, $n s$ not specified, $R P$ reversed-phase, $S G$ silica gel, $s p H P L C$ semi-preparative high-performance liquid chromatography, $T f a$ trifluoroacetic acid, Tet tetrahydrofuran, Wat water 
The first paper about CCC purification of PhGs from a whole plant crude extract proposed a two-phase solvent system composed of ethyl acetate-acetonitrile-water (2.6:2.6:4.8) in the reversed phase mode. When the system was run in the normal phase mode, addition of $2 \%$ methanol was required to adjust partition coefficients. Verbascoside and oraposide were successfully separated from Orobanche rapumgenistae Thuill. However, in both elution modes, the purities of the isolated PhGs did not exceed $82 \%$ (Viron et al. 1998). A subsequent report focused on CCC separation of verbascoside and 2'-acetylverbascoside from the stem extract of Cistanche salsa (C.A. Mey.) G. Beck (Lei et al. 2001a). As PhGs easily dissolve in $n$-butanol during liquid-liquid partitioning step before CCC, the authors decided to choose a biphasic solvent system containing equal amounts of $n$-butanol as organic phase modifier and ethanol as aqueous phase modifier (ethyl acetate- $n$-butanolethanol-water 4:0.6:0.6:5). Since the purity of verbascoside and $2^{\prime}$-acetylverbascoside isolated in the first run was not satisfactory, they were submitted to a second separation using the same solvent system (twostep CCC); consequently, the resulting PhGs had high purities $(>98 \%)$. As part of a more extensive study concerning the metabolic regulation of $\mathrm{PhGs}$ in the gastrointestinal tract, the same group of authors (Lei et al. 2001b) used a two-step CCC separation procedure to isolate verbascoside and isoverbascoside (purities higher than 95\%) from the methanolic extract of Beagle dogs faeces at $24 \mathrm{~h}$ after feeding them with $3 \mathrm{~g} / \mathrm{kg}$ PhGs from Cistanche tubulosa (Schrenk) Wight stems. The solvent systems used for this separation consisted of two different mixtures of ethyl acetate- $n$-butanol-ethanol-water $\quad(35: 6: 6: 50$ and 30:10:6:50). Injection of the $214 \mathrm{mg}$ sample into the first more non-polar system (36\% ethyl acetate- $6 \%$ butanol) caused a vast stripping of the stationary phase leaving only $16 \%$ of it in the column and resulting in co-elution of both target compounds. The second CCC run with a more polar solvent system (30\% ethyl acetate-10\% n-butanol) allowed a better separation. Similar systems (containing the same four solvents, but in different ratios) were employed by $\mathrm{Li}$ et al. (2008) with the aim to isolate verbascoside and other PhGs (2'-acetylverbascoside, cistanoside A, echinacoside, and isoverbascoside) from Cistanche deserticola Y.C. Ma. Han et al. (2012) used the same solvent system as in Lei et al. (2001a), but on a different CCC instrument and by adding a silica gel chromatography step before CCS. This allowed isolation of four PhGs from stem extract of Cistanche deserticola Y.C. Ma. When ethanol was replaced with methanol, new biphasic solvent systems were created, leading to CCC separation of rare $\mathrm{PhGs}$, such as: calcedariosides $\mathrm{A}$ and $\mathrm{B}$, as well as plantainosides $\mathrm{B}$ and $\mathrm{D}$ from Chirita longgangensis W.T. Wang, forsythoside A and suspensaside A from Forsythia suspensa (Thunb.) Vahl. (Duan et al. 2014; Yang et al. 2013). For the purification of verbascoside and isoverbascoside from Plantago psyllium L. seed extract, Li et al. (2005) successfully used the two phases of the mixture composed only of equal amounts of ethyl acetate and water; such a solvent system has the advantages of being easy to handle and having a quick settling time.

In most of the papers, single injections under isocratic conditions were enough to achieve $\mathrm{PhGs}$ in a purified form. However, in the case of salidroside from $R$. crenulata root extract, the fractions resulting from the first run with ethyl acetate- $n$-butanol-water (1:4:5) were subsequently eluted with an atypical solvent system composed of chloroform-methanolisopropanol-water (5:6:1:4) (Han et al. 2002). This type of chlorinated solvent systems is often used for the separation of root extracts in CCC, particularly ginsenosides (Skalicka-Wozniak and Garrard 2014). Working with chlorinated solvent systems is not a common practice in isolating PhGs by CCS. One reason is that the polarity of chloroform (or its replacement dichloromethane) does not provide a suitable range of partition coefficients for target PhGs. Also, with a move towards greener solvents, chlorinated solvent systems are less used due to health and safety restrictions. However, two examples of successful PhG separations have been reported. Chen et al. (2014) purified verbascoside and isoverbascoside from Pedicularis longiflora Rudolph var tubiformis (Klotz) Tsong with chloroform- $n$-butanol-methanolwater (4:3:4:5), whereas angoroside $C$ was isolated from Scrophularia ningpoensis Hemsl. with chloroform- $n$-butanol-methanol-water (4:1:3:2) (Tian et al. 2012).

In order to isolate PhGs, some authors added acetic acid to an ethyl acetate- $n$-butanol-water system. CCC separation is driven by partitioning of compounds between two liquid phases, similar to liquid-liquid extraction. The addition of a small amount of an organic acid can significantly improve the separation 
PhGs common CCC non-chlorinated solvent systems

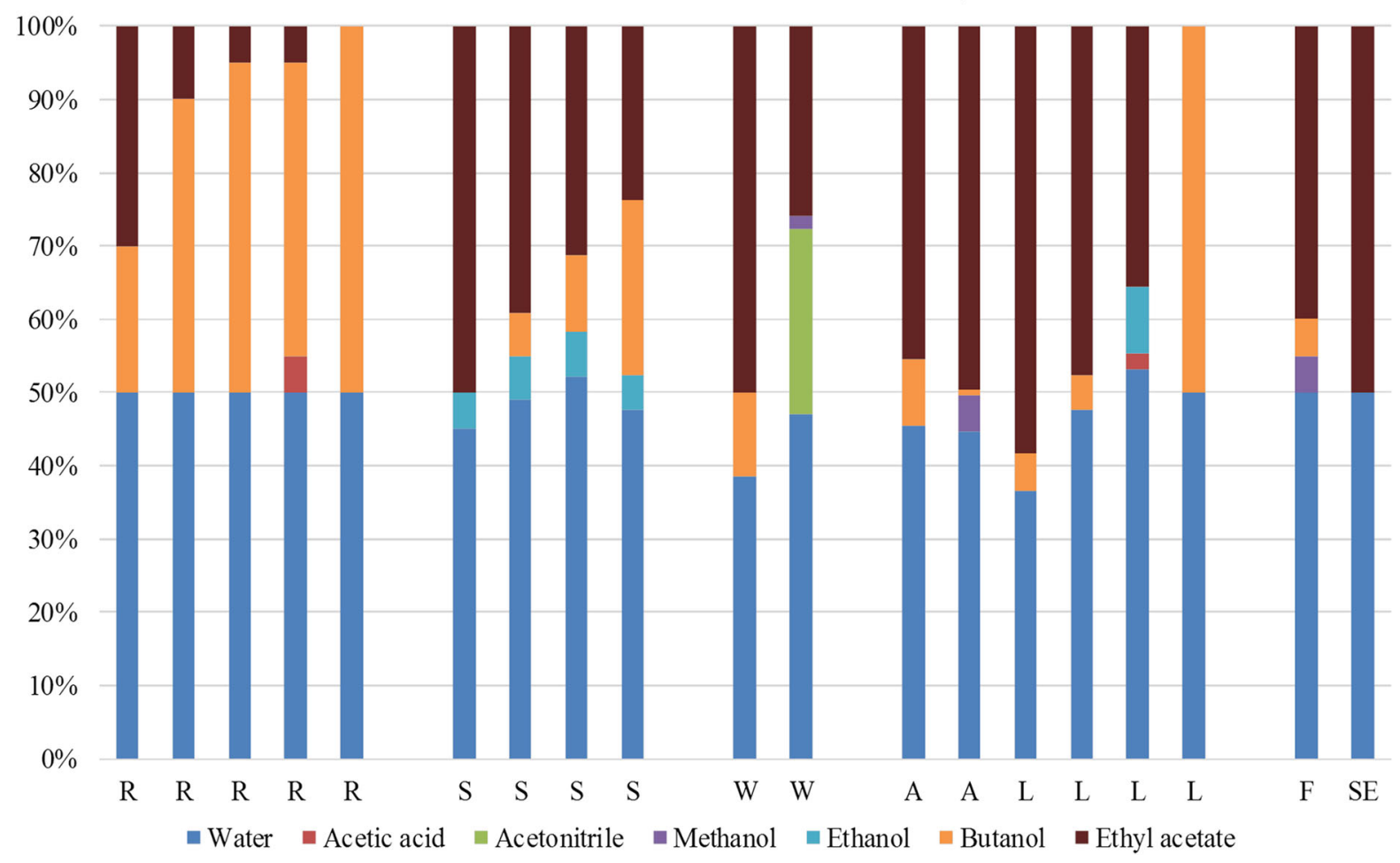

Fig. 3 Phenylethanoid glycosides-common CCC non-chlorinated solvent systems. $A$ aerial parts, $F$ fruits, $L$ leaves, $R$ roots, $S$ stem, $S E$ seeds, $W$ whole plant

by neutralizing the ionizable acidic functional groups and breaking hydrogen bonds (Berthod and Mekaoui 2011; Skalicka-Wozniak and Garrard 2014). Therefore, Xie et al. (2012b) obtained pure verbascoside and martynoside from Cistanche tubulosa (Schrenk) Wight with the help of ethyl acetate- $n$-butanolwater-glacial acetic acid (1:1.2:2:0.2), whereas Tong et al. (2009) isolated angoroside C from Scrophularia ningpoensis Hemsl. with ethyl acetate- $n$-butanolwater-glacial acetic acid (1:8:10:1). Another acidbased solvent system is represented by ethyl acetate$n$-butanol-water-glacial acetic acid (4:1:6:0.25) that led to the successful purification of forsythosides A and I from Forsythia suspensa (Thunb.) Vahl. (Sun et al. 2016). However, any addition of $\mathrm{pH}$ modifier should be carefully considered as it might lead to an additional processing step after CCC separation as well as compromise stability of target compounds.

Not all CCC separations required quaternary solvent mixtures. $n$-Butanol-water (1:1) system was successfully employed in the purification of verbascoside from Penstemon barbatus (Cav.) Roth and $P$. digitalis Nutt. ex Sims root and leaves extracts (Xie et al. 2010, 2012a; Wang et al. 2013). Additionally, this solvent system also led to the isolation of echinacoside from the same two species, but subsequent purification processes were needed. For the leaf extract, semi-preparative HPLC separation was performed (Wang et al. 2013), whilst for the root extract, the authors employed another CCC technique, known as recycling $\mathrm{CCC}$; echinacoside, with an advanced purity, was obtained from the 4th cycle (Xie et al. 2010). Recycling CCC is a repetitive (multiple) isocratic elution mode. It involves re-directing the eluent from the detector outlet back into the CCC column inlet (via pump inlet) at a set point of a separation. The mobile phase, which contains the target compounds, is introduced into the column inlet for the next separation cycle (Chen et al. 2011). Although recycling $\mathrm{CCC}$ methods require a prolonged time, the resolution of peaks is significantly improved, especially for compounds with similar K values.

It is worth mentioning, that as compared to other categories of natural constituents (e.g. coumarins) 
Iridoids common CCC solvent systems

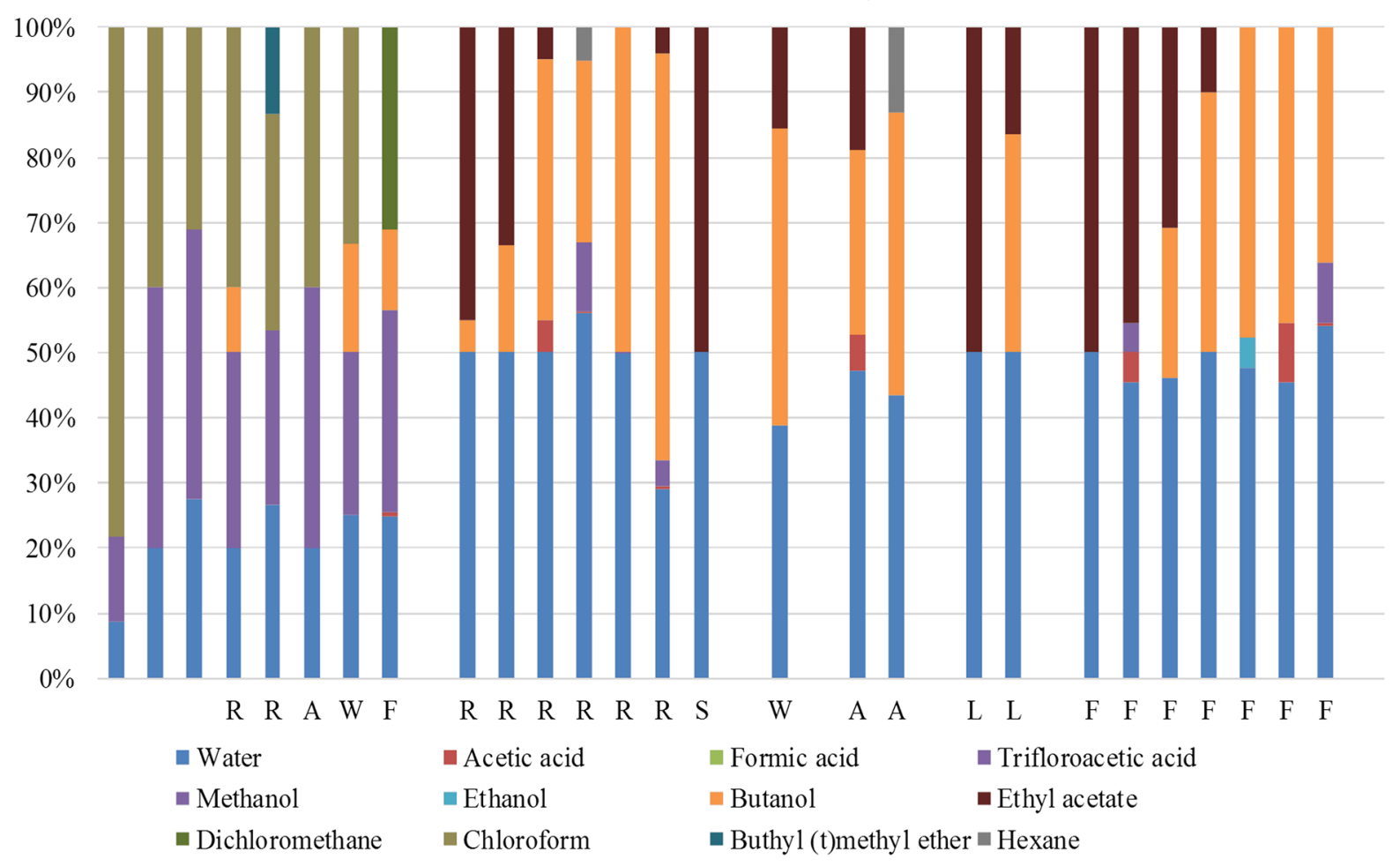

Fig. 4 Iridoids - common CCC solvent systems. $A$ aerial parts, $F$ fruits, $L$ leaves, $R$ roots, $S$ stem, $W$ whole plant

(Skalicka-Wozniak et al. 2014; Walasek et al. 2015) where crude extracts easily yielded pure compounds in a single CCS run, PhGs were rarely isolated directly from the raw extracts. Generally, after extraction with ethanol, methanol, ethanol-water or methanol-water mixtures, liquid-liquid partitioning extractions were conducted, and the ethyl acetate or $n$-butanol fractions were submitted to CCS directly or after column chromatography with appropriate solvents over silica gel or macroporous resins. These preliminary operations are needed to eliminate other undesired hydrophilic constituents and to make CCS more efficient. However, there are some examples when various PhGs were purified by CCS directly from the crude extracts. In the case of the methanolic extract of Abeliophyllum distichum Nakai leaves yielded high purity ( $>92.5 \%$ ) verbascoside, isoverbascoside, eutigoside $\mathrm{A}$ and cornoside ( $\mathrm{Li}$ et al. 2013). In addition, $70 \%$ ethanolic extract of a whole plant of Orobanche rapum-genistae Thuill. yielded verbascoside and oraposide having purities of $75 \%$ and $82 \%$, respectively (Viron et al. 1998).
Another approach to purify a crude plant extract is gradient elution. In CCC it can be a change in the mobile phase composition or flow rate, in a linear or step wise manner (Leitao et al. 2005; Ignatova et al. 2011). It also can be run in both reversed and normal phase mode. The main difference from HPLC is that in CCC any change of a mobile phase composition leads to a change in a stationary phase. Therefore, it is better to select a solvent system family where one of the phases' composition does not change much. This phase should be used as a stationary one (Berthod et al. 2002). Leitao et al. (2005) applied a step-gradient CCC method in normal phase mode with ethyl acetate- $n$-butanol-water (1:x:1, x =0.05-1) as solvent system for the fractionation of Stachytarpheta cayennensis (Rich.) Vahl. root extract. It produced three PhGs with a rhamnose unit in their structures (verbascoside, isoverbascoside and martynoside) and three iridoids, one of which was ipolamiide, recovered from the aqueous stationary phase at the extrusion stage. By applying the same gradient method, the separation of aerial part and leaf extracts of Lantana trifolia L. and Lippia alba $\mathrm{f}$. intermedia Moldenke (de 
Juliao et al. 2010; Leitao et al. 2015) required a modification of the solvent system by expanding its polarity range via addition of $n$-hexane, resulting thus in an $n$-hexane-ethyl acetate- $n$-butanol-water system (0.4:1:x:1, $\mathrm{x}=0.1-0.7)$. The ethyl acetate and water content range increased from 40-32 to 49-33\%, with $n$-butanol range reduced from $2-34$ to $4-23 \%$. The presence of $n$-hexane made the polarity difference between phases larger, as the organic phase became more non-polar. Once a method is set up, the gradient methodology can be repeated with other extracts, providing a reproducible elution of the same target compound under the same conditions (Leitao et al. 2015). It is the case for verbascoside invariably eluting at a $n$-butanol ratio of 0.5 (de Juliao et al. 2010). This step-gradient CCC system (0.4:1:x:1, $\mathrm{x}=0.1-0.7)$ was used for the separation of verbascoside and/or other PhGs, such as 2-(4-hydroxyphenyl)-ethyl- $O$ - $\beta$ D-glucopyranosyl-6- $O$ - $\beta$-D-glucopyranoside, $2^{\prime}$-apiosylverbascoside, isoverbascoside, allyssonoside, echinacoside, forsythoside $\mathrm{B}$, leucosceptoside $\mathrm{B}$, salidroside or wiedemannioside $\mathrm{C}$ from Lantana trifolia L. and Lippia alba f. intermedia Moldenke. In all these examples, the sample loading was in average $1 \mathrm{~g}$ per $100 \mathrm{~mL}$ column volume. Therefore, gradient elution in CCC might be used as a standard approach for fractionation of natural product extracts at all scales.

Several PhGs have been purified by CPC techniques in isocratic mode. CPC, due to its cascade mixing in consequtively connected cells (forming a separation column) and a single rotational axis, can retain solvent systems with a smaller density difference between liquid phases and can cope with emulsification. Therefore, much more polar additives like ethanol or acetone can be used in larger proportions, which causes longer settling times but brings the polarity difference between phases closer, resulting in the separation of compounds within a smaller polarity range. For example, Viron et al. (2000) repeated the separation of verbascoside and oraposide from Orobanche rapum-genistae Thuill. (Viron et al. 1998), by replacing acetonitrile with less toxic acetone and changing ratios of organic solvents resulting in an ethyl acetate-acetone-water (5:0.5:4.5) solvent system that allowed a higher loading of the crude extract. It seems the ethyl acetate-alcohol-water-based solvent systems, with 45-48\% ethyl acetate and water, can provide isolation of verbascoside from different extracts of upper part of a plant. Verbascoside was purified from Lippia citriodora Kunth with ethyl acetate-ethanol-water (5:0.5:4.5) (Cheimonidi et al. 2018) and Plantago asiatica L. with ethyl acetate- $n$ butanol-ethanol-water (5:5:1:10) (Li et al. 2009); the polarity of organic phase was adjusted by adding $n$ butanol. In the latter example, another PhG (plantamajoside) was isolated. Using $16 \%$ of tetrahydrofuran, the ethyl acetate-tetrahydrofuran-water (4.5:2:6) system provided the isolation of verbascoside in a single step, together with a large number of iridoids eluting from Capraria biflora L. (Lemus et al. 2015). Tetrahydrofuran and acetone have not been previously employed in CCC separations of PhGs.

\section{CCS and iridoids' isolation}

Of the 56 iridoids isolated through various CCS techniques from plants belonging mostly to Gentianaceae, Rubiaceae, Scrophulariaceae, Verbenaceae, Lamiaceae and Plantaginaceae families, 29 were iridoid glycosides, 19 secoiridoid glycosides, 5 bisiridoids and 3 non-glycosylated iridoids. Iridoid glycosides with a C-10 carbon skeleton and secoiridoids were present in crude extracts either together or separate, regardless the anatomical part of a plant used, whereas iridoid glycosides with a C-9 carbon skeleton were generally present in extracts on their own. Fruit extracts provided the largest number of iridoids isolated in one application when separated by CCC, followed by aerial parts, whole plants and roots. Gentiopicroside (10 times), sweroside (8 times) and swertiamarin (7 times) were the most frequently isolated compounds. Taking into consideration that iridoids are also water soluble compounds, ethyl acetate-alcohol-water solvent systems similar to those employed for the isolation of PhGs were used in the separation of this category of secondary metabolites, but generally with a higher content of $n$ butanol or other short-chain alcohols (Fig. 4). Ten out of 15 ethyl acetate-butanol-water systems had $35 \%$ butanol (or higher) and 40-50\% water.

Almost the same range of solvent systems were employed for root extracts separation to isolate iridoids as in the case of PhGs. Interestingly, eight chlorinated solvent systems were suggested mainly for secoiridoids purification. The polarity of the chloroform-methanol-water systems with $24 \%$ water in average and equal ratios of two organic solvent was 
adjusted by addition of $13 \% n$-butanol to make it more polar or $13 \% t$-butyl methyl ether to make it more apolar.

Mixtures of ethyl acetate- $n$-butanol-water (widely encountered in CCS of PhGs) led to the successful CCC purification of catalpol from Rehmannia glutinosa Libosch. (Tong et al. 2015), chlorotuberside, penstemonoside, phloyoside II and shanzhiside methyl ester from Lamiophlomis rotata (Benth.) Kudo (Yue et al. 2013b), geniposide and geniposidic acid from Gardenia jasminoides Ellis and Eucommia ulmoides Oliv. (Dai et al. 2013; Liang et al. 2014; Wang et al. 2015; Zhang et al. 2012; Zhou et al. 2005), gentiopicroside, loganic acid and swertiamarin from Gentiana scabra Bunge (Chen et al. 2017; Xu et al. 2007), harpagoside from Scrophularia ningpoensis Hemsl. (Tong et al. 2006) and ipolamiide from Stachytarpheta cayennensis (Rich.) Vahl. (Leitao et al. 2005). All previous compounds were isolated under isocratic conditions, except ipolamiide as a part of a large group for which a step-gradient elution was performed with ethyl acetate- $n$-butanol-water (1:x:1, $\mathrm{x}=0.05-1)$; this method allowed not only the isolation of ipolamiide and two other unidentified iridoid glycosides, but also of three PhGs mentioned above (verbascoside, isoverbascoside and martynoside). Addition of apolar ( $n$-hexane) or polar (ethanol) solvent to $n$-butanol-water system was also taken into consideration, depending on the structure of the target compound and matrix complexity. For instance, 6- $\beta$ hydroxygeniposide, gardenoside and geniposidic acid (C-10 iridoids) were isolated from Gardenia jasminoides Ellis fruit extract with the aid of $n$-butanolethanol-water (10:1:10) (Wang et al. 2015), whereas catalposide and verproside (C-9 iridoids) were purified from Veronica ciliata Fisch. aerial part extract with $n$ hexane- $n$-butanol-water (1.5:5:5) (Lu et al. 2016). In the former case, ethanol reduced the polarity difference between phases as it mainly goes into the aqueous phase making it more apolar; whereas the addition of $n$-hexane gives the opposite effect as it is not water miscible and it will be distributed in the organic phase only, making it more apolar. The simple solvent system ethyl acetate-water (1:1) was used for the CCC separation of two secoiridoids, ligstroside and oleuropein from Olea europea L. leaf extract. However, both compounds needed further semi-preparative HPLC purification (Zhang et al. 2014). Using the same solvent system, oleuropein was also isolated from Cynomorium songaricum Rupr. in a single step, with purity higher than $95 \%$ (Wang et al. 2016). The explanation might lie in the difference of the extract matrix as it was aqueous-ethanolic extract of leaves in Zhang et al. (2014) and aqueous stem extract in Wang et al. (2016). Systems from traditional $n$-hexane-ethyl acetate-methanol-water family (called HEMWat) have been rarely used for the CCC separation of water soluble compounds such as iridoids due to systems polarity range. However, there is one example when $n$ hexane-ethyl acetate-methanol-water $(1: 3: 1: 3)$ was used for purification of trifloroside, a less polar secoiridoid, from the ethyl acetate root extract of Gentiana scabra Bunge (Chen et al. 2017). Some similar compounds were isolated using solvent systems containing chloroform. For example, gentiopicroside was separated from Gentiana scabra Bunge with chloroform-tert-butyl methyl ether-methanolwater (5:2:4:4) (Wang et al. 2007), Gentiana macrophylla Pallas and Swertia franchetiana $\mathrm{H}$. Smith with chloroform-methanol-water (4:4:2) (Cheng et al. 2010; Huangfu et al. 2007) and Swertia mussotii Franch with chloroform- $n$-butanol-methanol-water (Chen et al. 2015). Moreover, harpagoside was isolated from Scrophularia ningpoensis Hemsl. with chloroform- $n$-butanol-methanol-water $\quad$ (4:1:3:2) (Tian et al. 2012) and isovogeloside and vogeloside from Halenia campanulata Cuatrec. with chloroformmethanol-water (9:12:8) (Hostettmann and Marston 2001).

Optimizing partitioning of ionized compounds or depressing emulsification of a solvent system with the help of small amounts of organic acids is a very common practice in the CCC purification of iridoids. Acidic modifiers can substantially shorten the settling time of the chosen biphasic solvent system, improve the retention of the stationary phase and therefore improve separation, especially when the sample solution contains acidic impurities. Moreover, due to protonation, the molecules that contain ionizing groups (carboxylic, phenolic) become more hydrophobic and therefore their affinity for the organic phase is increased (Ito 2005). For example, mixtures of ethyl acetate- $n$-butanol-water containing acetic acid led to the isolation of harpagoside from Scrophularia ningpoensis Hemsl. (Tong et al. 2009), deglucoserrulatoside, gentiopicroside, loganic acid, sweroside and swertiamarin from Gentiana macrophylla Pall. (Wu et al. 2012). Ethyl acetate- $n$-butanol- 
methanol-1\% (aq) acetic acid (0.5:7.5:0.5:3.5) was successfully used as solvent system for the separation of five iridoid glycosides $\left(6^{\prime}-O-\beta\right.$-D-glucopyranosyl gentiopicroside, gentiopicroside, loganic acid, sweroside and swertiamarin) from the traditional Tibetan medicine Gentiana crassicaulis Duthie ex Burk in a single run (Liang et al. 2013b). Moreover, some acidified systems with nonpolar solvents ( $n$-hexane or dichloromethane) were also employed with the purpose to isolate similar plant secondary metabolites. $n$ Hexane- $n$-butanol-methanol- $0.4 \%$ (aq) acetic acid (1.4:8:3:15.5) led to the one-step purification of 8-hydroxy-10-hydrosweroside, swertiamarin and trifloroside from Gentiana scabra Bunge (Liang et al. 2007), whereas dichloromethane- $n$-butanol-methanol-water-glacial acetic acid (5:2:5:4:0.1) to that of sweroside, morronoside and loganin from Cornus officinalis Sieb. et Zucc. (Liang et al. 2013a). Another variation of an acidified solvent system tested for the separation of the latter two compounds from the same plant species consisted of $n$-butanol-methanol-1\% (aq) acetic acid (4:1:6) (Liu et al. 2009). A mixture of $n$-butanol-0.1\% (aq) trifluoroacetic acid (1:1) was successfully employed for the isolation of loganic acid, gentiopicroside and swertiamarin from Gentiana macrophylla Pall. under consecutive flow rate gradient conditions (from 1.5 to $5 \mathrm{~mL} / \mathrm{min}$ ) (Rho et al. 2016).

A very peculiar example is represented by the isolation of picrosides I-III from the crude ethanolic extract of Picrorhiza scrophulariiflora Pennel. when three different CCC techniques were applied. First, the crude extract was eluted under isocratic conditions with $n$-hexane-ethyl acetate-methanol-water $(1: 2: 1: 2)$. Then the main fraction was subjected to elution-extrusion CCC (EECCC) with ethyl acetaten-butanol-water-concentrated formic acid (4:1:5:0.005) when picroside II and another fraction resulted. EECCC was implemented by Berthod et al. (2003) to extend the hydrophobicity window of the classical CCC, resulting in the extrusion of the most retained solutes out of the column with acceptable peak resolution. The method has two steps: the first step is a classical CCC elution, whereas in the next one, the stationary phase containing the partially separated hydrophobic solutes is extruded out of the column in a continuous way using the liquid stationary phase. EECCC can be time and solvent sparing, being advantageous for retrieving compounds with high $\mathrm{K}$ values which normally take a long time to elute but may be already separated within the column. Eventually, the impure fraction previously obtained by EECCC was submitted to CECCC with ethyl acetate-water (1:1) when pure picrosides I and III were achieved in the 6th cycle (Chen et al. 2011).

In the study of Liang et al. (2018), 18 compounds were isolated from Cornus officinalis Sieb. et Zucc. fruit extract, of which 15 iridoids C-10 and secoiridoids. Kingiside, morroniside, loganic acid, swertiamarin, 10-hydroxyhastatoside, 10-hydroxycornin, 7-dehydrologanin, sweroside were separated with $n$ butanol-water-glacial acetic acid (5:5:1), whereas for the isolation of 10-hydroxycornin and loganin, the elution was changed to EECCC mode. 7-O-(4maloyl)loganin, cornusides I and II, $7(S)$ and $7(R)$ $O$-ethylmorroniside and sarracenin were purified with the help of ethyl acetate-methanol-water-glacial acetic acid (5:0.5:5:0.5). Such a high concentration of glacial acetic acid in the $n$-butanol-water system was required due to a high loading of the crude, which was $1 \mathrm{~g}$ on $300 \mathrm{~mL} \mathrm{CCC} \mathrm{column,} \mathrm{to} \mathrm{ensure} \mathrm{constant}$ $\mathrm{pH}$. Also, for such separations, CCC often run in reversed elution mode for better $\mathrm{pH}$ control. Beside the great number of compounds isolated practically with only two different solvent systems, it is also noticeable that the CCC equipment was not coupled with a classical UV detector, but with a mass spectrometer (that has a higher sensitivity). Moreover, most of these compounds are very rare iridoids, while 7-O-(4-maloyl)loganin was a new characterized constituent.

As compared to PhGs, when very few compounds were separated with $\mathrm{CPC}$, this technique was more often employed for isolating iridoids (8 papers out of 11 found for the CPC part of the review). In three applications, CPC was practically used for the production of iridoids from large quantities of extracts isolating seven C-9 iridoids from the aerial part extract (Lemus et al. 2015), five secoiridoids from another aerial part extract (Mandova et al.2017) and four bissecoiridoids and two secoiridoids from a whole plant extract (Lehbili et al. 2018) with an average loading of $1.3 \mathrm{~g}$ per every $100 \mathrm{~mL}$ of column volume in normal phase elution mode.

In the study of Markovic et al. (2014), $23.9 \mathrm{~g}$ of aucubin was purified from $105 \mathrm{~g}$ of leaf extract from Aucuba japonica Thunb. using ethyl acetate- $n$ propanol-water $(7: 3: 10)$ in three repetitive runs. This solvent system, but with slightly higher $n$-propanol 
content, was later successfully used by the same research group twice more to separate C-9 iridoids. In Lemus et al. (2015), two new (3-hydroxymyopchlorin and 5-hydroxyglutinoside) and five known C-9 iridoids (caprarioside, macfadyenoside, 8- $O$-acetylharpagide, catalpol and 6- $\beta$-hydroxyantirrhide) were separated from Capraria biflora L. The methanolic extract of aerial parts was submitted to CPC, first with tetrahydrofuran-ethyl acetate-water (4:9:12) and second with ethyl acetate- $n$-propanol-water (8:4:12). Adjusting the solvent ratios to ethyl acetate- $n$ propanol-water (3:2:5) allowed the purification of 8- $O$-acetylharpagide from the aqueous leaf extract of Oxera coronata Kok in one run (Remeur et al. 2017). Replacing $n$-propanol with its isomer, as in ethyl acetate-2-propanol-water (3:2:5), led to the isolation of C-10 iridoid geniposide from Gardenia jasminoides Ellis (Kim and Kim 2007). Increasing the ethyl acetate content from 30 to $48 \%$ and using ethanol (19\%) instead of 2-propanol (20\%) made this solvent system less polar. The polarity of the final solvent system ethyl acetate-ethanol-water (7.5:3:5) was enough to enrich the main target, swertiamarin, and separate four more secoiridoids (gentiopicroside, secologanol, secoxyloganin, sweroside) from the aqueous extract of Centaurium erythraea Rafn. aerial parts (Mandova et al. 2017).

Two new bis-secoiridoids (7- $O$-( $E$-p-coumaroyl)sylvestroside I, 7-O-(E-caffeoyl)-sylvestroside I) together with two other known bis-secoiridoids and three secoiridoids (sylvestroside I, septemfidoside, sweroside, eustomoside and eustomoruside) were isolated from Scabiosa stellata Cav. with less polar solvents of tert-butyl methyl ether-acetonitrile-water (3:3:4) (Lehbili et al. 2018). This type of solvent systems seems working well for less polar iridoids. Loganetin, a non-glycosidic iridoid, was separated from the stem bark extract of Alstonia scholaris (L.) R.Br. with tert-butyl methyl ether-acetonitrile-water $(3: 1.5: 3)$ in which $\mathrm{pH}$ modifiers were added to both phases $(\mathrm{HCl}$ in the lower aqueous phase and triethylamine in the upper organic phase) (Maurya et al. 2014). The conditions are typical for $\mathrm{pH}$-zone refining elution mode, which is often used for separation of ionized compounds (Ito 2005). However, the solvent system was equilibrated before sample injection resulting in running the $\mathrm{CPC}$ separation in isocratic normal phase mode with rather unusual approach to $\mathrm{pH}$ control. Another example of a less polar solvent system was used for purification of oleuropein from Olea europea L. with $n$-hexane-ethyl acetate-ethanol-water (1:9:1:9) (Boka et al. 2015). In all large scale separations described above, the CPC fractions needed further purification steps with the help of conventional chromatographic methods.

Similar to PhGs, isolation of pure iridoids directly from the crude extract after a single CCC run is unusual, but not impossible. Gentiopicroside, $6^{\prime}-O-\beta$ D-glucopyranosyl gentiopicroside, loganic acid, sweroside and swertiamarin were isolated in pure form after one $\mathrm{CCC}$ experiment directly from the $57.5 \%$ ethanolic extract of Gentiana crassicaulis Duthie ex Burk. obtained by microwave-assisted extraction. As it can be easily deduced, the composition of the microwave-assisted extraction solvent was not chosen randomly, but after optimization experiments in which parameters such as the effect of ethanol concentration, irradiation time and microwave power on the yield of target compounds were statistically analyzed. Consequently, it is understandable why only one purification step was needed for the isolation of pure iridoids (Liang et al. 2013b).

Biological relevance of phenylethanoid glycosides and iridoids isolated through CCC

PhGs and iridoids have never ceased to surprise researchers in terms of their potential medicinal uses, as they were found to possess a broad spectrum of bioactivities (antioxidant, neuroprotective, antitumor, antimicrobial, anti-inflammatory, immunomodulating, cardioprotective, etc.). Numerous review articles have focused on summarizing the pharmacological activities of these two classes of phytochemicals (Dinda et al. 2007b, 2009, 2011; Fu et al. 2008; Tundis et al. 2008; Xue and Yang 2016). However, in this section the emphasis will be put only on those few papers dealing with both CCS and biological evaluation of isolated compounds.

It was interesting to notice that, although the first PhGs/iridoids isolated through CCS date from 1998, the earliest reports that included at least one biological assay were published in 2010, with an increased frequency of papers describing bioactivity screenings in the last few years. This tendency might have its reasoning in the fact that researchers were initially engaged mostly in proving that CCS is a powerful alternative tool to conventional chromatographic 
techniques. Once more and more papers showed that CCS is indeed fast and efficient in isolating PhGs and iridoids, the efforts were readily focused on showing the pharmacological utility of the purified compounds, which is practically the purpose of any isolation in natural product research.

In the case of PhGs, only 6 papers addressed not only the CCS process, but also testing the antioxidant, sedative, aldose reductase inhibition and cytotoxic properties of the isolated compounds. Several PhGs were assessed in various antioxidant tests. Verbascoside and isoverbascoside from Pedicularis longiflora Rudolph var. tubiformis (Klotz.) Tsoong exhibited strong 2,2-diphenyl-1-pycrylhydrazyl (DPPH) radical scavenging activity, with $\mathrm{IC}_{50}$ values of 15.6 and $18.9 \mu \mathrm{g} / \mathrm{mL}$, respectively; the activity was slightly lower than that of a very well-known natural antioxidant, gallocatechin $\left(\mathrm{IC}_{50}=7.2 \mu \mathrm{g} / \mathrm{mL}\right)($ Chen et al. 2014). Salidroside, together with the new PhG (2(4-hydroxyphenyl)-ethyl- $O-\beta$-D-glucopyranosyl-6- $O$ $\beta$-D-glucopyranoside) from Rhodiola crenulata Fish et Mey, showed moderate DPPH scavenging effects with $\mathrm{IC}_{50}$ values of $10.97 \mu \mathrm{M}$ and $12.13 \mu \mathrm{M}$, respectively (Chen et al. 2012). Duan et al. (2014) investigated the antioxidant capacity of PhGs from Chirita longgangensis W.T.Wang. All compounds showed scavenging effects in different systems, but the potency was different, depending on the reactive oxygen species to be quenched. In the hydroxyl radical-, superoxide anion- and hydrogen peroxide-luminol chemiluminescence assays, the sequence of scavenging effects was as follows: plantanoiside $\mathrm{D}>$ plantainoside $\mathrm{B}>$ calcedarioside $\mathrm{A}>$ calcedarioside $\mathrm{B}$; plantanoiside $\mathrm{B}>$ calcedarioside $\mathrm{B}>$ plantainoside $\mathrm{D}>$ calcedarioside $\mathrm{A}$; and calcedarioside $\mathrm{B}>$ plantainoside B $>$ plantainoside D $>$ calcedarioside A. The PhGs isolated from Abeliophyllum distichum Nakai (verbascoside, isoverbascoside, eutigoside $\mathrm{B}$ and cornoside) were tested in a 2,2'-azino-bis(3ethylbenzothiazoline-6-sulphonic acid) (ABTS) scavenging assay. The compounds exerted their antioxidant activity in the following order: verbascoside $>$ isoverbascoside $\gg$ eutigoside $\mathrm{B}>$ cornoside; verbascoside was by far the most active constituent, with $\mathrm{IC}_{50}$ value lower even than that of the positive control quercetin ( $\mathrm{Li}$ et al. 2013). Moreover, in the same study, the four PhGs were submitted to an aldose reductase inhibition assay. The inhibition of enzyme decreased in the order verbascoside $>$ isoverbascoside $>$ eutigoside $\quad \mathrm{B} \gg$ cornoside (Li et al. 2013).

de Juliao et al. 2010 investigated the sedative effects of two PhGs from Lantana trifolia L. (verbascoside and betonyoside F). The reason why only these two PhGs were submitted to further biological analyses was probably dictated by the yielded amounts: a few mg of samioside and martynoside vs. hundreds of $\mathrm{mg}$ of betonyoside $\mathrm{F}$ and verbascoside. However, as the yield of verbascoside was around four times higher than that of betonyoside $\mathrm{F}$, only verbascoside was included in an in vivo experiment. It was shown that the ethanolic extract, ethyl acetate extract and verbascoside produced an intense reduction of the walked squares at 1 and $10 \mathrm{mg} / \mathrm{kg}$ (extracts) and 1 and $3 \mathrm{mg} /$ $\mathrm{kg}$ (verbascoside) in one and $2 \mathrm{~h}$ after the administration in mice, respectively. However, verbascoside at $300 \mu \mathrm{M}$ had no affinity for the $\left[{ }^{3} \mathrm{H}\right]$ flunitrazepam binding sites, whereas betonyoside $\mathrm{F}$ showed an $\mathrm{IC}_{50}$ of $550 \mu \mathrm{M}$. These values were found to be very high compared to the activity of classical benzodiazepines ( $\mathrm{IC}_{50}$ values in $\mathrm{nM}$ range), suggesting that this is probably not the mechanism by which verbascoside exerts its sedative properties.

The cytotoxic potential of verbascoside purified from Lippia citriodora Kunth by CPC was investigated by Cheimonidi et al. (2018). It was shown that verbascoside produced a significant toxicity in metastatic mouse skin carcinoma A5 cells, mouse melanoma B16.F1 and B16.F10 cells, osteosarcoma U2 OS, Sa OS and KH OS cells; moreover, it exhibited synergistic effects with hydrogen peroxide, doxorubicin and epoxomicin and re-sensitized doxorubicin resistant osteosarcoma cell lines. It was suggested that verbascoside exerted its cytotoxicity by inhibiting protein kinase $\mathrm{C}$ (enzyme that affects the signaling status of many oncogenic pathways) and modulating antioxidant responses, proteostatic modules and immune responses. Additionally, intraperitoneal administration of verbascoside decreased the tumor growth in an in vivo melanoma mouse model, by activating anti-tumor-reactive immune responses; however, oral administration was not efficient.

There are only eight papers dealing with both CCS and evaluation of biological activity of isolated iridoids. Chen et al. (2017) investigated the anti-nitric oxide production effects of four iridoids isolated from Gentiana scabra Bunge. The results showed that all compounds were able to effectively inhibit nitric oxide 
production induced by lipopolysaccharide (200 ng/ $\mathrm{mL}$ ) in a dose-dependent manner in murine microglial BV-2 cells. At $250 \mu \mathrm{M}$, gentiopicroside, loganic acid, swertiamarin and trifloroside produced an inhibition of $73.3 \%, 58.1 \%, 40.8 \%$ and $41.0 \%$, respectively.

Some of the CCS isolated iridoids have been proven to possess potent antioxidant activity in various assays. For instance, oleuropein from Cynomorium songaricum Rupr. exerted promising DPPH radical scavenging activity (Wang et al. 2016), whereas catalposide and verproside from Veronica ciliata Fisch. showed good ferric reducing antioxidant power, DPPH and ABTS cation radicals scavenging effects (Lu et al. 2016). Moreover, the latter two iridoids were evaluated in the same study for their anti-hepatocarcinoma activity using HepG2 cells. These compounds inhibited the proliferation of tumor cells in a concentration-dependent manner, with $\mathrm{IC}_{50}$ values of $184.59 \mu \mathrm{g} / \mathrm{mL}$ for catalposide and $177.147 \mu \mathrm{g} / \mathrm{mL}$ for verproside. All 15 iridoids isolated from Cornus officinalis Sieb. et Zucc. were investigated for their cytotoxic activity against glioma U87MG and LN229 cells, but no effects were detected at $100 \mu \mathrm{M}$ after 5 days of incubation (Liang et al. 2018).

Of the seven iridoids isolated from Capraria biflora L. through CPC, the two new compounds (3-hydroxymyopochlorin and 5-hydroxyglutinoside) and caprarioside were subjected to in vitro screening against a panel of 37 bacterial strains; however, no activity was detected at $50 \mathrm{mg} / \mathrm{L}$ (Lemus et al. 2015). Nevertheless, even if some iridoids are considered inefficient as antibacterial agents, Maurya et al. (2014) investigated the synergistic action of loganetin and nalidixic acid against Escherichia coli nalidixic acid resistant and sensitive strains. When tested alone, loganetin was found to be inactive as it displayed a MIC value of $500 \mu \mathrm{g} / \mathrm{mL}$. But when $10 \mu \mathrm{g} / \mathrm{mL}$ of loganetin was mixed with nalidixic acid, the MIC of nalidixic acid was reduced by $4-8$ times. Therefore, the suggested bioenhancing potential of loganetin can be useful in lowering the dose of antibiotics, reducing the drug resistance development frequency and increasing the efficacy of antibiotics against the multidrug resistant E. coli strains.

It is worth to note that among these papers, only two described the bioactivity-guided isolation of various iridoids. The $70 \%$ ethanolic extract of Scabiosa stellata Cav. was initially fractionated over Diaion HP-20 and the five yielded fractions were then screened for antimicrobial activity against 22 microorganisms and anti-tyrosinase activity. As two fractions (B and C) were proven to have superior biological properties, they were both subsequently submitted to CPC experiments. Twelve compounds were purified, of which seven iridoids (two new and five known). 7-O-(E-p-Coumaroyl)- and 7-O-(E-caffeoyl)-sylvestroside I showed good inhibitory effects against Enterococcus faecalis (MIC $=31.2 \mu \mathrm{g} / \mathrm{mL}$ ), Staphylococcus epidermidis ( $\mathrm{MIC}=31.2 \mu \mathrm{g} / \mathrm{mL}$ ) and $S$. aureus $(\mathrm{MIC}=62.5 \mu \mathrm{g} / \mathrm{mL}$ ). Eustomoruside exhibited good inhibitory effects against $E$. faecalis and $S$. aureus ( $\mathrm{MIC}=62.5 \mu \mathrm{g} / \mathrm{mL}$ ). Only sylvestroside I showed antibacterial activity against $E$. coli in addition to $S$. aureus (MIC $=62.5 \mu \mathrm{g} / \mathrm{mL}$ ). All isolated iridoids were also evaluated for their DPPH radical scavenging effect, but only eustomoruside and eustomoside were active, exhibiting $\mathrm{IC}_{50}$ values of 7.1 and $7.2 \mu \mathrm{g} / \mathrm{mL}$, respectively, whereas for the other compounds the 50\% DPPH inhibition could not be reached even at $200 \mu \mathrm{g} / \mathrm{mL}$. In the anti-tyrosinase assay, none of the compounds was active at the concentration of $665 \mu \mathrm{g} / \mathrm{mL}$. The cytotoxic activity of the previously unknown iridoids was tested in vitro against HT1080 fibrosarcoma cell line and only 7-O-(E-caffeoyl)sylvestroside $\mathrm{I}$ was active, with an $\mathrm{IC}_{50}$ value of $35.9 \pm 0.06 \mu \mathrm{g} / \mathrm{mL}$ (Lehbili et al. 2018).

Zhang et al. (2014) developed a bioassay-guided isolation of pure constituents from Olea europea L. using an on-line HSCCC method coupled with a postcolumn $\alpha$-amylase anti-diabetic assay. The six fractions purified from olive leaves on resin AB-8 were initially screened for their anti-diabetic effects. As fraction IV showed the strongest inhibitory activity, it was further submitted to HSCCC separation with ethyl acetate-water $(1: 1)$ and then $\alpha$-amylase was added to each sample collection tube behind the HSCCC column. Finally, the fractions showing anti-diabetic activity were subsequently purified by semi-preparative HPLC, yielding oleuropein and ligstroside. The anti-diabetic activity of oleuropein and ligstroside was not further investigated.

\section{Conclusions}

All the above presented examples show the enormous potential of CCS in isolating and purifying PhGs and iridoids from different plant sources with various two- 
phase solvent systems. In most of the cases, CCS was efficiently integrated into the separation workflow of these secondary metabolites. Up-stream (liquid-liquid extraction, column chromatography over macroporous resins, polyamide, Sephadex LH-20 or silica gel) and down-stream (semi-preparative HPLC, column chromatography over silica gel or Sephadex LH20) purification techniques were sometimes needed. Nevertheless, CCS offers removal of a complex matrix of natural extracts making further processing easier and more effective, as well as the possibility of creating single-step isolation of a target constituent (e.g. verbascoside from Abeliophyllum distichum Nakai, gentiopicroside from Gentiana crassicaulis Duthie ex Burk).

Different solvent systems can be successfully used for the separation of the same compounds (e.g. verbascoside), while a proper selected solvent system can be modulated to target similar constituents from the same extract (e.g. iridoids from Cornus officinalis Sieb. et Zucc., phenylethanoids from Cistanche deserticola Y.C. Ma). It is also important to notice that CCS functions as a highly versatile preparative method, as different operation (normal and reversedphase) and elution (isocratic, step-gradient, elutionextrusion, cycling-elution, flow-enhanced) modes can be readily employed. The examples thoughtfully described in the current review may be used as starting point for CCS users on their sinuous road of isolating known or waiting to be discovered PhGs and iridoids.

Open Access This article is distributed under the terms of the Creative Commons Attribution 4.0 International License (http:// creativecommons.org/licenses/by/4.0/), which permits unrestricted use, distribution, and reproduction in any medium, provided you give appropriate credit to the original author(s) and the source, provide a link to the Creative Commons license, and indicate if changes were made.

\section{References}

Alipieva K, Korkina L, Orhan IE, Georgiev MI (2014) Verbascoside: a review of its occurrence, (bio)synthesis and pharmacological significance. Biotechnol Adv 32:1065-1076

Berthod A, Mekaoui N (2011) Distribution ration, distribution constant and partition coefficient. Countercurrent chromatography retention of benzoic acid. J Chromatogr A 1218:6024-6030

Berthod A, Brown L, Leitao GG, Leitao SG (2002) Countercurrent chromatography the support free liquid stationary phase. Chapter 2-operating a countercurrent chromatography machine. In: Barcelo D, Berthod A (eds) Comprehensive analytical chemistry, vol 38, 1st edn. Elsevier, New York, pp 21-47

Berthod A, Ruiz-Angel MJ, Carda-Broch S (2003) Elution-extrusion countercurrent chromatography. Use of the liquid nature of the stationary phase to extend the hydrophobicity window. Anal Chem 75:5886-5894

Boka VI, Argyropoulou A, Gikas E et al (2015) Employment of high-performance thin-layer chromatography for the quantification of oleuropein in olive leaves and the selection of a suitable solvent system for its isolation with centrifugal partition chromatography. Planta Med $81: 1628-1635$

Boros CA, Stermitz FR (1990) Iridoids. An updated review. Part I. J Nat Prod 53:1055-1147

Boros CA, Stermitz FR (1991) Iridoids. An updated review. Part II. J Nat Prod 54:1173-1246

$\mathrm{Bu}$ Z, He Q, Zhao R et al (2017) Separation and purification of acteoside from Rehmannia glutinosa by combining macroporous resin with high-speed countercurrent chromatography. Se Pu 35:1014-1021

Calis I (2002) Biodiversity of phenylethanoid glycosides. In: Sener B (ed) Biodiversity. Springer, Boston, pp 137-149

Cheimonidi C, Samara P, Polychronopoulos P et al (2018) Selective cytotoxicity of the herbal substance acteoside against tumor cells and its mechanistic insights. Redox Biol 16:169-178

Chen Z, Wu J, Shen W et al (2011) Counter-current chromatographic method for preparative scale isolation of picrosides from traditional Chinese medicine Picrorhiza scrophulariiflora. J Sep Sci 34:1910-1916

Chen D, Fan J, Wang P et al (2012) Isolation, identification and antioxidative capacity of water-soluble phenylpropanoid compounds from Rhodiola crenulata. Food Chem 134:2126-2133

Chen C, Zhao XH, Yue HL et al (2014) Separation of phenylpropanoid glycosides from a Chinese herb by HSCCC. J Chromatogr Sci 52:395-399

Chen C, Chen T, Liu Y et al (2015) Rapid screening, identification, separation and purification of four bioactive compounds from Swertia mussotii Franch. Sep Sci Technol 50:604-610

Chen B, Peng Y, Wang X et al (2017) Preparative separation and purification of four glycosides from Gentianae radix by high-speed counter-current chromatography and comparison of their anti-NO production effects. Molecules 22:2002

Cheng HY, Li T, Du YZ et al (2010) Separation and preparation of gentiopicroside and sweroside from the extracts of $S w$ ertia franchetiana $\mathrm{H}$. Smith by high speed counter-current chromatography. J Anhui Agric Sci 1:38-40

Dai X, Huang Q, Zhou B et al (2013) Preparative isolation and purification of seven main antioxidants from Eucommia ulmoides Oliv. (Du-zhong) leaves using HSCCC guided by DPPH-HPLC experiment. Food Chem 139:563-570

de Juliao LS, Leitao SG, Lotti C et al (2010) Flavones and phenylpropanoids from a sedative extract of Lantana trifolia L. Phytochemistry 71:294-300

Dinda B, Debnath S, Harigaya Y (2007a) Naturally occurring iridoids. A review, part 1. Chem Pharm Bull 55:159-222 
Dinda B, Debnath S, Harigaya Y (2007b) Naturally occurring secoiridoids and bioactivity of naturally occurring iridoids and secoiridoids. A review, part 2. Chem Pharm Bull 55:689-728

Dinda B, Roy Chowdhury D, Mohanta BC (2009) Naturally occurring iridoids, secoiridoids and their bioactivity. An updated review, part 3. Chem Pharm Bull 57:765-796

Dinda B, Debnath S, Banik R (2011) Naturally occurring iridoids and secoiridoids. An updated review, part 4. Chem Pharm Bull 59:803-833

Duan W, Bai A, Lin X et al (2014) Isolation and purification of highly polar antioxidants from Chirita longgangensis by combination of macroporous resin and HSCCC. Chromatographia 77:707-713

El-Naggar LJ, Beal JL (1980) Iridoids. A review. J Nat Prod 43:649-707

Friesen JB, Pauli GF (2007) Rational development of solvent system families in counter-current chromatography. J Chromatogr A 1151:51-59

Friesen JB, McAlpine JB, Chen SN, Pauli GF (2015) Countercurrent separation of natural products: an update. $\mathrm{J}$ Nat Prod 78:1765-1796

Fu G, Pang H, Wong YH (2008) Naturally occurring phenylethanoid glycosides: potential leads for new therapeutics. Curr Med Chem 15:2592-2613

Geu-Flores F, Sherden NH, Courdavault V et al (2012) An alternative route to cyclic terpenes by reductive cyclization in iridoid biosynthesis. Nature 492:132-142

Han X, Zhang T, Wei Y et al (2002) Separation of salidroside from Rhodiola crenulata by high-speed counter-current chromatography. J Chromatogr A 971:237-241

Han L, Ji L, Boakye-Yiadom M, Li W, Song X, Gao X (2012) Preparative isolation and purification of four compounds from Cistanche deserticola Y.C. Ma by high-speed counter-current chromatography. Molecules 17:8276-8284

Hopmann E, Frey A, Minceva M (2012) A priori selection of the mobile and stationary phase in centrifugal partition chromatography and counter-current chromatography. J Chromatogr A 1238:68-76

Hostettmann K, Marston A (2001) Countercurrent chromatography in the preparative separation of plant-derived natural products. J Liq Chromatogr Rel Technol 24:1711-1721

Huangfu ZK, Ni SF, Sun WJ (2007) Preparative separation of gentiopicroside from aerial part of Gentiana macrophylla Pall by high speed counter-current chromatography. Chin J Anal Chem 35:883-886

Ignatova S, Sutherland I (2015) The 8th international conference on counter-current chromatography held at Brunel University, London, UK, July 23-25, 2014. J Chromatogr A 1425:1-7

Ignatova S, Sumner N, Colclough N, Sutherland IA (2011) Gradient elution in counter-current chromatography: a new layout for an old path. J Chromatogr A 1218:6053-6060

Ito $\mathrm{Y}$ (2005) Golden rules and pitfalls in selecting optimum conditions for highspeed counter-current chromatography. J Chromatogr A 1065:145-168

Jimenez C, Riguera R (1994) Phenylethanoid glycosides in plants: structure and biological activity. Nat Prod Rep 11:591-606
Kim CY, Kim J (2007) Preparative isolation and purification from Gardenia fruits by centrifugal partition chromatography. Phytochem Anal 18:115-117

Lehbili M, Alabdul Magid A, Hubert J et al (2018) Two new bisiridoids isolated from Scabiosa stellata and their antibacterial, antioxidant, anti-tyrosinase and cytotoxic activities. Fitoterapia 125:41-48

Lei L, Yang F, Zhang T et al (2001a) Preparative isolation and purification of acteoside and $2^{\prime}$-acetyl acteoside from Cistanches salsa (C.A. Mey) G. Beck by high-speed counter-current chromatography. J Chromatogr A 912:181-185

Lei L, Yang F, Zhang T et al (2001b) Preparative isolation and purification of phenylethanoid glycosides from the extract of faeces of beagle dogs by high-speed countercurrent chromatography. J Liq Chrom Rel Technol 24:2187-2195

Leitao GC, de Souza PA, Moraes AA, Brown L (2005) Stepgradient CCC separation of phenylpropanoid and iridoid glycosides from roots of Stachytarpheta cayennensis (Rich.) Vahl. J Liq Chrom Rel Technol 28:2053-2060

Leitao GC, Pinto SC, de Oliveira DR et al (2015) Gradient x isocratic elution $\mathrm{CCC}$ on the isolation of verbascoside and other phenylethanoids: influence of the complexity of the matrix. Planta Med 81:1609-1613

Lemus C, Grougnet R, Ellong EN et al (2015) Phytochemical study of Capraria biflora L. aerial parts (Scrophulariaceae) from Martinique island (French West Indies). Phytochem Lett 13:194-199

Li HB, Chen F (2001) Preparative isolation and purification of salidroside from the Chinese medicinal plant Rhodiola sachalinensis by high-speed counter-current chromatography. J Chromatogr A 932:91-95

Li L, Tsao R, Liu Z et al (2005) Isolation and purification of acteoside and isoacteoside from Plantago psyllium L. by high-speed counter-current chromatography. J Cromatogr A 1063:161-169

Li L, Tsao R, Yang R et al (2008) Isolation and purification of phenylethanoid glycosides from Cistanche deserticola by high-speed counter-current chromatography. Food Chem 108:702-710

Li L, Liu C, Chen Z et al (2009) Isolation and purification of plantamajoside and acteoside from plant extract of Plantago asiatica L. by high-performance centrifugal partition chromatography. Chem Res Chin Univ 25:817-821

Li HM, Kim JK, Jang JM et al (2013) Analysis of the inhibitory activity of Abeliophyllum distichum leaf constituents against aldose reductase by using high-speed counter current chromatography. Arch Pharm Res 36:1104-1112

Liang $\mathrm{Y}, \mathrm{Hu} \mathrm{J}$, Chen $\mathrm{H}$ (2007) Preparative isolation and purification of four compounds from Chinese medicinal herb Gentiana scabra Bunge by high-speed countercurrent chromatography. J Liq Chromatogr Rel Technol 30:509-520

Liang J, He J, Zhu S et al (2013a) Preparation of main iridoid glycosides in Fructus corni by macroporous resin column chromatography and countercurrent chromatography. J Liq Chromatogr Rel Technol 36:983-999

Liang J, Ito Y, Zhang X et al (2013b) Rapid preparative separation of six bioactive compounds from Gentiana crassicaulis Duthie ex Burk. using microwave-assisted 
extraction coupled with high-speed counter-current chromatography. J Sep Sci 36:3934-3940

Liang Z, Yang M, Xu X et al (2014) Isolation and purification of geniposide, crocin-1, and geniposidic acid from the fruit of Gardenia jasminoides Ellis by high-speed counter-current chromatography. Sep Sci Technol 49:1427-1433

Liang J, Qiao B, Syed N, Sun W (2018) A mass spectrometry guided elution-extrusion counter-current chromatography protocol for isolation of eighteen terpenoids from Fructus corni and assessment of their anti-glioma activities. Microchem J 137:464-472

Lindner S, Geu-Flores F, Brase S et al (2014) Conversion of substrate analogs suggest a Michael cyclization in iridoid biosynthesis. Chem Biol 21:1452-1456

Liu L, Sun A, Wu S, Liu R (2009) Preparative purification of morroniside and loganin from Fructus corni by combination of macroporous absorption resin and HSCCC. J Chromatogr Sci 47:333-336

Lu Q, Sun Y, Shu Y et al (2016) HSCCC separation of two iridoid glycosides and three phenolic compounds from Veronica ciliata and their in vitro antioxidant and antihepatocarcinoma activities. Molecules 21:1234

Mandova T, Audo G, Michel S, Grougnet R (2017) Off-line coupling of new generation centrifugal partition chromatography device with preparative high pressure liquid chromatography-mass spectrometry triggering fraction collection applied to the recovery of secoiridoid glycosides from Cenaurium eyrthraea Rafn. (Gentianaceae). J Chromatogr A 1513:149-156

Markovic D, Barboux C, de Chiu YC et al (2014) Centrifugal partition chromatography: an efficient tool to access highly polar and unstable synthetic compounds on a large scale. RSC Adv 4:63254

Marston A, Hostettmann K (2006) Developments in the application of counter-current chromatography to plant analysis. J Chromatogr A 1112:181-194

Maurya A, Dwivedi GR, Darokar MP, Srivastava SK (2014) Preparative isolation of bioenhancer loganetin from $\mathrm{Al}$ stonia scholaris by fast centrifugal partition chromatography. Sep Sci Technol 49:773-777

Mudge E, Lopes-Lutz D, Brown PN, Schieber A (2012) Purification of phenylalkanoids and monoterpene glycosides from Rhodiola rosea L. roots by high-speed countercurrent chromatography. Phytochem Anal 24:129-134

Oyourou JN, Combrinck S, Regnier T, Marston A (2013) Purification, stability and antifungal activity of verbascoside from Lippia javanica and Lantana camara leaf extracts. Ind Crops Prod 43:820-826

Qiu HY, Fan WX, Fu P et al (2013) General acteoside of Rehmanniae leaves in the treatment of primary chronic glomerulonephritis: a randomized controlled trial. Afr J Tradit Complement Altern Med 10:109-115

Qiu HY, Fu P, Fan WX et al (2014) Treatment of primary chronic glomerulonephritis with Rehmannia glutinosa acteosides in combination with the angiotensin receptor blocker irbesartan: a randomized controlled trial. Phytother Res 28:132-136

Remeur C, Le Borgne E, Gauthier L et al (2017) HPLC-ELDS quantification and centrifugal partition chromatography isolation of 8-O-acetylharpagide from Oxera coronate (Lamiaceae). Phytochem Anal 28:242-246
Rho T, Jung M, Lee MW et al (2016) Efficient methods for isolating five phytochemicals from Gentiana macrophylla using high-performance countercurrent chromatography. J Sep Sci 39:4723-4731

Sampaio-Santos MI, Kaplan MAC (2001) Biosynthesis significance of iridoids in chemosystematics. J Braz Chem Soc 12:144-153

Schlauer J, Budzianowski J, Kukulczanka K, Ratajczak L (2004) Acteoside and related phenylethanoid glycosides in Byblis liniflora Salisb. plants propagated in vitro and its systematic significance. Acta Soc Bot Pol 73:9-15

Skalicka-Wozniak K, Garrard I (2014) Counter-current chromatography for the separation of terpenoids: a comprehensive review with respect to the solvent systems employed. Phytochem Rev 13:547-572

Skalicka-Wozniak K, Mroczek T, Koziol E (2014) High-performance countercurrent chromatography separation of Peucedanum cervaria fruit extract for the isolation of rare coumarin derivatives. J Sep Sci 38:179-186

Sun Y, Hou Z, Liu Z, Wang J (2016) Ionic liquid-based ultrasonic-assisted extractin of forsythosides from the leaf of Forsythia suspensa (Thunb.) Vahl and subsequent separation and purification by high-speed counter-current chromatography. J Chromatogr Sci 54:1445-1452

Tian J, Ye X, Shang Y et al (2012) Preparative isolation and purification of harpagoside and angoroside $\mathrm{C}$ from the root of Scrophularia ningpoensis Hemsley by high-speed counter-current chromatography. J Sep Sci 35:2659-2664

Tong S, Yan J, Lou J (2006) Preparative isolation and purification of harpagoside from Scrophularia ningpoensis Hemsley by high-speed counter-current chromatography. Phytochem Anal 17:406-408

Tong S, Sheng L, Yan J, Lou J (2009) Preparative separation of phenylpropanoid glycoside from Scrophularia ningpoensis Hemsley by high speed countercurrent chromatography and ESI-MS ${ }^{\text {n }}$ analysis. J Liq Chromatogr Rel Technol 32:2322-2333

Tong S, Chen L, Zhang Q et al (2015) Separation of catalpol from Rehmannia glutinosa Libosch. by high-speed countercurrent chromatography. J Chromatogr Sci 53:725-729

Tundis R, Loizzo MR, Menichini F et al (2008) Biological and pharmacological activities of iridoids: recent developments. Mini Rev Med Chem 8:399-420

Viljoen A, Mncwangi N, Vermaak I (2012) Anti-inflammatory iridoids of botanical origin. Curr Med Chem 19:2104-2127

Viron C, Lhermite S, Andre P, Lafosse M (1998) Isolation of phenylpropanoid glycosides from Orobanche rapum by high speed countercurrent chromatography. Phytochem Anal 9:39-43

Viron C, Pennanec R, Andre P, Lafosse M (2000) Large scale centrifugal partition chromatography in purification of polyphenols from Orobanche rapum. J Liq Chromatogr Rel Technol 23:1681-1688

Walasek M, Grzegorczyk A, Malm A, Skalicka-Wozniak K (2015) Bioactivity-guided isolation of antimicrobial coumarins from Heracleum mantegazzianum Sommier \& Levier (Apiaceae) fruits by high-performance countercurrent chromatography. Food Chem 186:133-138

Wang YY, Wang YP, Liu JY et al (2007) Preparative separation of gentiopicrin from Gentiana scabra bunge by high speed 
counter current chromatography. Zhong Yao Cai 30:789-791

Wang Q, Xie J, Fang S et al (2013) Complementary application of HSCCC and semi-preparative HPLC for rapid separation of phenylethanoid glycosides from Penstemon digitalis. Chin Herb Med 5:280-285

Wang Y, Chen Y, Deng L et al (2015) Systematic separation and purification of iridoid glycosides and crocetin derivatives from Gardenia jasminoides Ellis by high-speed countercurrent chromatography. Phytochem Anal 26:202-208

Wang G, Huang X, Pei D et al (2016) DPPH-HPLC-DAD analysis combined HSCCC for screening and identification of radical scavengers in Cynomorium songaricum Rupr. New J Chem 40:3885

Wu W, Ye H, Tang M et al (2012) Using high-performance counter-current chromatography combined with preparative high performance liquid chromatogramphy for the separation of bioactive compounds from the water extract of Gentiana macrophylla Pall. Sep Sci Technol 47:762-768

Xie J, Deng J, Tan F, Su J (2010) Separation and purification of echincoside from Penstemon barbatus (Can.) Roth by recycling high-speed counter-current chromatography. J Chromatogr B 878:2665-2668

Xie J, Tan F, Zhu J et al (2012a) Separation, purification and quantification of verbascoside from Penstemon barbatus (Cav.) Roth. Food Chem 135:2536-2541

Xie J, Xu X, Liu Q et al (2012b) Isolation and purification of echinacoside and acteoside from Cistanche tubulosa (Schrenk) Wight by high-speed counter-current chromatography. J Liq Chromatogr Rel Technol 35:2602-2609

Xu YW, Qi Y, Han X et al (2007) Preparative separation of gentiopicrin from Radix Gentianae by high-speed countercurrent chromatography with macroporous resin. Zhongguo Zhong Yao Za Zhi 32:2595-2597
Xue Z, Yang B (2016) Phenylethanoid glycosides: research advances in their phytochemistry, pharmacological activity and pharmacokinetics. Molecules 21:991

Yang M, Xu X, Xie C et al (2013) Isolation and purification of forsythoside A and suspensaside from Forsythia suspensa by high-speed counter-current chromatography. J Liq Chromatogr Rel Technol 36:2895-2904

Yue HL, Zhao XH, Mei LJ, Shao Y (2013a) Separation and purification of five phenylpropanoid glycoside from Lamiophlomis rotata (Benth.) Kudo by a macroporous resin column combined with high-speed counter-current chromatography. J Sep Sci 36:3123-3129

Yue HL, Zhao XH, Wang QL, Tao YD (2013b) Separation and purification of water-soluble iridoid glucosides by high speed counter-current chromatography combined with macroporous resin column separation. J Chromatogr B 936:57-62

Zhang M, Ignatova S, Hu P et al (2012) Cost-efficient and process-efficient separation of geniposide from Gardenia jasminoides Ellis by high-performance counter-current chromatography. Sep Purif Technol 89:193-198

Zhang J, Huang X, Sun X et al (2014) Efficient method for the screening and identification of anti-diabetic components in the leaves of Olea europea L. New J Chem 38:3796

Zhang L, Yue HL, Zhao XH et al (2015) Separation of four phenylpropanoid glycosides from a Chinese herb by HSCCC. J Chromatogr Sci 53:860-865

Zhou T, Fan G, Hong Z et al (2005) Large-scale isolation and purification of geniposide from the fruit of Gardenia jasminoides Ellis by high-speed counter-current chromatography. J Chromatogr A 1100:76-80

Publisher's Note Springer Nature remains neutral with regard to jurisdictional claims in published maps and institutional affiliations. 